\title{
Definition of a Merging Assistant Strategy Using Intelligent Vehicles
}

Riccardo Scarinci (corresponding author)

Centre for Transport Studies

Dep. of Civil, Environmental \& Geomatic Engineering

University College London

Gower Street, WC1E 6BT London, United Kingdom

Tel. +44 (0) 2076791590

Fax +44 (0) 2076793042

email riccardo.scarinci.09@ucl.ac.uk

Andreas Hegyi

Dep. of Transport \& Planning

Fac. of Civil Engineering \& Geosciences

Delft University of Technology

P.O. Box 5048, 2600 GA Delft, The Netherlands

Tel. +31 (15) 2789644

Fax +31 (15) 2783179

email a.hegyi@tudelft.nl

Benjamin Heydecker

Centre for Transport Studies

Dep. of Civil, Environmental \& Geomatic Engineering

University College London

Gower Street, WC1E 6BT London, United Kingdom

Tel. +44 (0) 2076791553

Fax +44 (0) 2076793042

email b.heydecker@ucl.ac.uk

\section{Abstract}

In the area of active traffic management, new technologies provide opportunities to improve the use of current infrastructure. Vehicles equipped with in-car communication systems are capable of exchanging messages with the infrastructure and other vehicles. This new capability offers many opportunities for traffic management. This paper presents a novel merging assistant strategy that exploits the communication capabilities of intelligent vehicles. The proposed control requires the cooperation of equipped vehicles on the main carriageway in order to create merging gaps for on-ramp vehicles released by a traffic light. The aim is to reduce disruptions to the traffic flow created by the merging vehicles. This paper focuses on the analytical formulation of the control algorithm, and the traffic flow theories used to define the strategy. The dynamics of the gap formation derived from theoretical considerations are validated using a microscopic simulation. The validation indicates that the control strategy mostly developed from macroscopic theory well approximates microscopic traffic behaviour. The results present encouraging capabilities of the system. The size and frequency of the gaps created on the main carriageway, and the space and time required for their creation are compatible with a real deployment of the system. Finally, we summarise the results of a previous study showing that the proposed merging strategy reduces the occurrence of congestion and the number of late-merging vehicles. This innovative control strategy shows the potential of using intelligent vehicles for facilitating the merging manoeuvre through use of emerging communications technologies.

Keywords Intelligent Transport system (ITS), Active Traffic Management (ATM), Intelligent Vehicle, Motorway Merging, Ramp Metering, Cooperative Vehicle. 


\section{Introduction}

Emerging communication technologies open new management possibilities in the field of Intelligent Transport Systems (ITS). Particularly promising are technologies allowing vehicles to infrastructure (V2I) and vehicle to vehicle $(\mathrm{V} 2 \mathrm{~V})$ communication. This new communication capability could enable cooperation among vehicles, opening a new research thread known as Cooperative ITS (NEARCTIS, 2009; Netten et al., 2011; COOPERS, 2010). Using cooperation, the current ITS can be improved, and a new generation of Intelligent Transport Systems can be developed. It is following these new challenges and opportunities that the present research takes place.

This paper proposes a merging assistant strategy for on-ramps using intelligent vehicles capable of V2I communication. The innovative strategy, called Cooperative Merging Assistant (CoopMA), rearranges vehicles on the main carriageway to create large gaps for facilitating the merging of on-ramp traffic. We introduce the control algorithm and present the governing equations based on both macroscopic and microscopic traffic flow theories. The control strategy describes the size, frequency, space and time needed to create useful gaps for merging. Then, we use microscopic simulation as "ground truth" to validate the gap formation dynamic derived by the theoretical considerations. This paper focuses on the algorithm definition. However, we report a brief summary of the traffic performance based on previous work for completeness (Scarinci et al., 2015).

Although researchers and practitioners have given much attention to the use of emerging technologies applied to ITS, new possibilities and challenges are constantly opening. For this reason, this research area is still largely unexplored and more studies are needed to understand all the possible opportunities given by these technological advances. The current study adds another input into the field of Cooperative ITS, suggesting an innovative application able to optimise further the use of motorways.

The paper is structured as follows. Section 2 reviews active traffic management algorithms for on-ramp merging using intelligent vehicles. The Cooperative Merging Assistant strategy is described in Section 3. We first introduce the control strategy, then, we present the equations governing the algorithm. The analytical formulation is validated against microscopic simulation in Section 4. A brief evaluation of the Cooperative Merging Assistant system traffic performance is given in Section 5. Finally, Section 6 summarises the main conclusions.

\section{Literature review}

A vast literature on merging assistant strategies is present. It ranges from the first work on autonomous vehicles on motorways, e.g. Mammano and Bishop (1992), to recent work on cooperative vehicles, e.g. Xie et al. (2016). We refer to Scarinci and Heydecker (2014) for a complete review of the control algorithms, evaluation methods and optimization strategies.

In the following, we review three main categories of control algorithms for facilitating motorway on-ramp merging. Each category uses a different type of intelligent vehicles to manage the merging process.

(i) Automated vehicles. Completely automated vehicles are capable to perform longitudinal and lateral movements without driver's interventions. In the 1990s, Yang et al. (1993) Yang and Kurami (1993) and Kachroo and Li (1997) started developing control algorithms with the main goal of providing a smooth merging. This is achieved by guiding on-ramp vehicles into existing gaps on the main carriageway. Antoniotti et al. (1997) and Ran et al. (1999) further extend the control capability of the merging procedure controlling also vehicles traveling on the main carriageway. First field tests are performed by Kato et al. (2002) and $\mathrm{Lu}$ et al. (2004). These tests evaluate both the technological requirement for communication and control and the theoretical algorithm capabilities. More recently, Marinescu et al. (2010) (2012) popose a centralized algorithm that detects when a control intervention is needed. Automated vehicles, when needed, are guided to slots available for merging. Xie et al. (2016) propose a merging control strategy optimizing the speed profile of vehicles close to the on-ramp. The authors also develop a simulation platform for testing and evaluating control strategies for cooperative vehicles.

(ii) Cooperative Adaptive Cruise Control. Vehicles equipped with Cooperative Adaptive Cruise Control (CACC) can maintain a fixed gap with the preceding vehicle and receive information to proactively change speed. Uno et al. (1999) suggest to place a "virtual vehicle" in front of a vehicle traveling in the main carriageway to create a suitable gap for merging vehicles. The impact of using CACC for facilitating merging in mixed traffic is evaluated by Xu and Sengupta (2003) and Davis (2007). In their tests, equipped vehicles on the main carriageway create merging gaps for on-ramp vehicles. In another 
study on mixed traffic, van Arem et al. (2006) evaluate the impact of CACC in lane changing manoeuvres. Also Pueboobpaphan et al. (2010) propose a merging assistant strategy for mixed traffic. The proposed algorithm aims to increase traffic flow stability. Wang et al. (2007) and Kanavalli et al. (2008) apply "proactive merging strategy" to merging algorithms. Thanks to the communication capability, intelligent vehicles can collect data of the surroundings vehicles and adjusting their speed.

(iii) On-board display. Vehicles equipped with an on-board display can receive suggestions of speed or lane changes from the infrastructure to which the driver should react. This topic is more recent, and less research has been done. Park et al. (2011) identify vehicles on the main carriages that performing a lane-change can facilitate the merging of on-ramp vehicles. The lane-changing suggestion, if performed, should be able to reduce merging conflicts. Daamen et al. (2011) test the traffic impacts of sending messages to single vehicles in case of a large speed difference between two vehicles is present, and a platoon conflicts with a merging vehicle.

The majority of the authors evaluate the performance of the presented algorithms using a microscopic simulation. The key performance indicators evaluated, such as gap creation dynamics, merging speed and total delay, show how the control strategies improve the traffic flow.

We now identify where the proposed Cooperative Merging Assistant (CoopMA) strategy is located within the review literature. The CoopMA strategy requests the cooperation of intelligent vehicles on the main carriageway. Instead, the on-ramp vehicles are controlled by a traffic light, as done by the ramp metering system (Papageorgiou and Kotsialos, 2002). Only one intelligent vehicle on the main carriageway is requested at each traffic cycle. The innovation is the complete use of the existing ramp metering infrastructure, i.e. the traffic light and detector loops, and the extend of the management capability to intelligent vehicles equipped with a communication device on the main carriageway. The details of the control strategy are presented in the next section.

\section{Cooperative Merging Assistant control strategy}

This section introduces the Cooperative Merging Assistant (CoopMA) system and describes the procedure adopted to derive the analytical formulation of the control strategy based on a combination of macroscopic and microscopic theory of traffic flow. Section 3.1 gives an overview of the CoopMA. The assumptions and limitations are discussed in Section 3.2. Then, the complete formulation of the CoopMA control algorithm is presented in Section 3.3 and discussed in Section 3.4.

\subsection{Cooperative Merging Assistant system}

Before introducing the CoopMA control strategy, it is convenient to summarise the reasoning behind the development of this system. The algorithm presented by this research tries to address a limitation of the current ramp metering system and to take advantage of the opportunities given by emerging technology. A significant limitation of ramp metering (RM) is that, although the RM aims to operate the entire junction as a controllable system, only the on-ramp vehicles are regulated. No control is made on the main carriageway flow in order to facilitate the merging process. The on-ramp vehicles may perturb the main carriageway flow in the merging area, and due to the presence of a traffic light, the merging manoeuvres became even more difficult (Zheng and McDonald, 2007). This phenomenon is more pronounced if, instead of one single vehicle, a platoon of vehicles is released (Kotsialos et al., 2006; Papageorgiou and Papamichail, 2008; Hegyi, 2004) as in the case of Full Traffic Cycle release algorithm. The perturbation is mainly created by vehicles that during the merging manoeuvre are not able to find a suitable gap. These vehicles, often referred to as late merging, are forced to decrease their speed while approaching the end of the acceleration lane. The late merging vehicles will then merge with low speeds and accept smaller gaps, disrupting the main carriageway vehicles. If this phenomenon triggers a traffic breakdown, the motorway capacity is affected and therefore, the maximum flow is reduced. Leclercq et al. (2011) describe the behaviour of the merging vehicles as moving bottlenecks responsible for the capacity drop at merging, identifying the difference in speed between the merging vehicles and the main carriageway traffic as the principal cause. The proposed Cooperative Merging Assistant strategy is based on these considerations.

The basic idea of CoopMA is to coordinate the release of on-ramp vehicles with gaps on the main carriageway created for facilitating the merging. These gaps can be created by rearranging the position of the vehicles present on the near-side lane, i.e. the lane close to the on-ramp. The vehicles on the nearside lane are induced to move in platoons with a higher density. This densification is done by collecting empty spaces to create useful gaps. The rearrangement is done by reducing the speed of an intelligent 

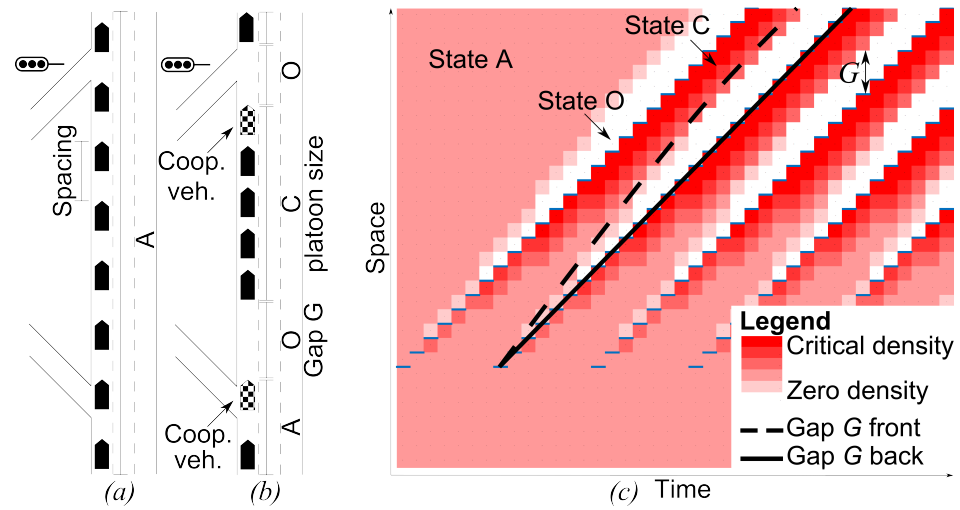

Figure 1: Conceptual representation of the effects on traffic flow under Cooperative Merging Assistant. (a) vehicle configuration if CoopMA is not applied, and (b) if CoopMA is applied with creation of platoons and gaps. (c) representation of the spatio-temporal evolution of the main carriageway vehicle density with formation and evolution of gaps $G$ suitable for merging due to the decrease in speed of the cooperative vehicles. Adapted from (Scarinci et al., 2015).

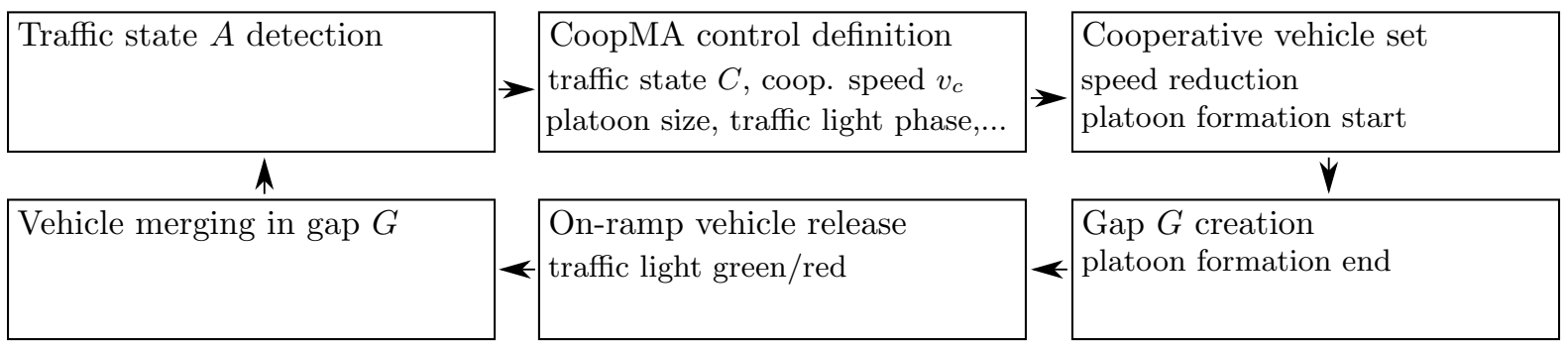

Figure 2: Cooperative Merging Assistant control strategy logical representation and main steps.

vehicle on the main carriageway. The vehicle speed can be reduced either automatically, transmitting instructions to the vehicle Cruise Control, or manually, showing a message on the on-board display and requesting an action from the driver. Because the behaviour of this specific vehicle aims to facilitate others to its own detriment, it is henceforth referred to as cooperative vehicle.

Figure 1 presents a conceptual representation of the CoopMA logic. Figures 1(a) shows the vehicle configuration in the uncontrolled scenario. In this case, the vehicles are travelling at a traffic state $A$, and the gaps among them follow a random distribution. For example, in congested situations it has been estimated to have an average of 1.5 seconds with a standard deviation of 0.4 second (Banks, 2003). Instead, Figure 1(b) shows the rearranged vehicle configuration when the flow is controlled with CoopMA. The vehicles are compacted in state $C$. Due to the cooperative vehicle reduced speed, they travel in a platoon formation followed by an empty space $G$, defined as state $O$. Figure $1(c)$ represents the traffic density on the main carriageway when CoopMA is applied. Once the cooperative vehicle is set, the formation of the gap $G$ begins. The gap starts gradually to expand while moving downstream. Meanwhile, the upstream vehicles, due to the slow vehicle in front, compact behind the cooperative vehicle to a more dense traffic state, state $C$. Subsequently, another cooperative vehicles are set cyclically. The gap $G$ is represented in Figure 1(b) as the distance between the front of the cooperative vehicle and the rear of the last vehicle of the platoon downstream, and as an area of zero density, State $O$, in Figure 1(c). This figure also presents the space-time dynamic of the upstream and downstream fronts of the gap $G$. The upstream-front is defined by the cooperative vehicle trajectory in the spatio-temporal diagram. While the downstream-front dynamic is delimited by the trajectory of the first vehicle downstream from the cooperative vehicle, i.e. the last vehicle in the platoon. Knowing the spatio-temporal dynamic of the gap $G$ and the travel time from the traffic signal to the merging location, it is possible to calculate the phases of the on-ramp traffic light. The cycle should be chosen to ensure the coordination between the release of on-ramp vehicles and the gap $G$. Meanwhile the green length, i.e. the number of on-ramp vehicles for every traffic light cycle, should be proportional to the gap size. Figure 2 summarises the main steps of the CoopMA logic. 


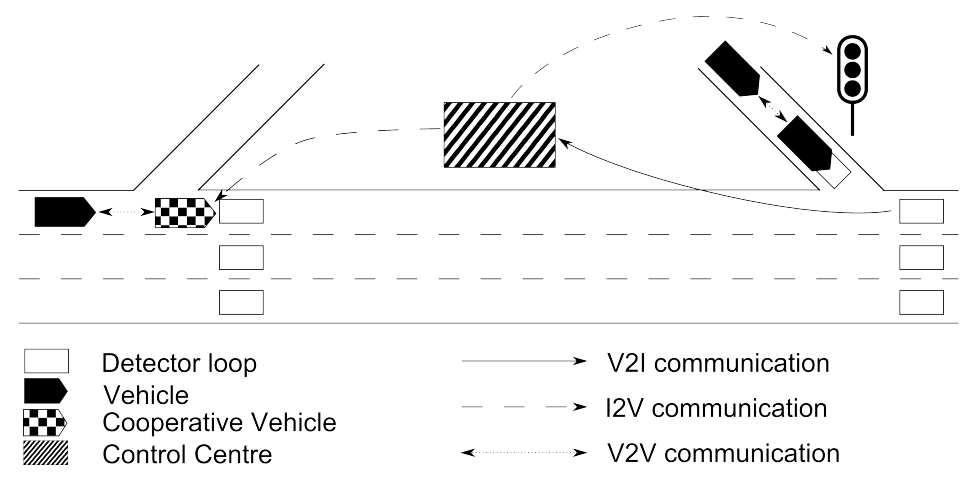

Figure 3: Representation of the communication request by the Cooperative MA system. The control centre estimates the traffic conditions on the on-ramp and main carriageway, releases the on-ramp vehicles using the traffic signal and slows down the cooperative vehicle on the main carriageway.

The CoopMA system requires the following exchange of information (represented graphically in Figure 3$)$ :

- Vehicle to Infrastructure (V2I). On-ramp and main carriageway vehicles provide information on the traffic state to the control centre. This communication can be done using the same detector loops already present in junctions equipped with traditional RM. Therefore, no equipped vehicles are necessary for this communication, although floating car data provided by intelligent vehicles can be integrated for a better estimation of the traffic states.

- Infrastructure to Vehicle (I2 V). The control centre releases the on-ramp vehicles and slows down cyclically the cooperative vehicles. For the release of on-ramp vehicles, the CoopMA uses the traffic light already present in junctions equipped with RM. Therefore, no intelligent vehicles are required. On the other hand, vehicles equipped with on board communication system are necessary for creating the main carriageway gap $G$.

We note that Vehicle to Vehicle (V2V) communication is not necessary, because it is implemented in an indirect way. Except the cooperative vehicles and the first vehicle released by the traffic light (both directly controlled by the CoopMA algorithm), the movements of the other vehicles are restricted by normal car following rules. Therefore, $\mathrm{V} 2 \mathrm{~V}$ communication is not required, although any type of V2V communicant could improve the system. From the description of the necessary communication, it is clear that the CoopMA needs a moderate presence of advanced technology, requiring only one intelligent vehicle per traffic light cycle. Moreover, the system has been designed to make full use of the traditional RM installation and be integrated with the RM control strategy. The maximum number of vehicles $n_{\mathrm{o}}$ that can merge in the gap $G$, as calculated by the CoopMAcan be used by the traditional RM control policy as a further constraint for the determination of the target on-ramp flow. The traditional RM control policy defines the on-ramp flow to maintain the optimal traffic condition based on the traffic state, and use $q_{\mathrm{o}}^{\max }$ as upper limit. The traditional ramp metering control policy defines the target on-ramp flow and the associated length of the green phase $C_{g}$, while the Cooperative Merging Assistant provides gaps for better merging, and defines the traffic light cycle $C_{c}$ in order to coordinate on-ramp vehicles with the gap $G$.

\subsection{Assumptions and limitations}

The CoopMA control strategy is based mainly on macroscopic considerations of traffic flow. Therefore, a series of assumptions and limitations are made to derive the governing equations described in the next section.

We assume homogeneous drivers and vehicles, and the control laws are based on the average behaviour. For example, an average vehicle acceleration is used, and no intra-vehicle variability is considered. All vehicles need the same amount of time to merge, and platoon of vehicles require the same gap than the sum of individual vehicles. Although only one intelligent vehicle on the main carriageway is requested at each traffic cycle, we assume that all vehicles may be cooperative vehicles, i.e. $100 \%$ penetration rate. As a consequence, all main carriageway vehicles may be able to cooperate. Although 
it is possible to extend the control strategy to mixed traffic, the following of this paper is based on this assumption.

We assume that the gap created is preserved. The presence of cooperative vehicles traveling at a low speed could trigger additional lane changing behaviour of the upstream vehicles trying to overtake them. In order to preserve the gap created, these induced movements should be considered and limited. Off-side lane changes, i.e. from the near-side (slow lane) towards the off-side (fast lane), can be allowed for any vehicle except the cooperative ones. This type of lane change increases the gap available for merging, although the consequences on platoon formation should be evaluated carefully. Instead, to protect the gap created, near-side lane changes should be restricted in the vicinity of the on-ramp. This restriction could be enforced applying one-side solid line marking on the road surface. We assume full compliance of the drivers to these rules. For the definition of the control strategy equations, we assume that the cooperative vehicles reduce their speed instantaneously. This assumption is discussed in the following of the paper.

The CoopMA strategy has several limitations. The system operates only in the free-flow state of the fundamental diagram (speed higher that the critical speed). If the motorway is in congested state, other ITS should be used to recover to the free-flow state. The CoopMA is derived mainly from macroscopic traffic flow theory, as commonly seen in traditional ramp meeting and variable speed limit control strategies Papageorgiou and Papamichail (2008); Hegyi et al. (2008). As a result, the strategy does not use optimization algorithms to define the control actions. Moreover, model predictive control (MPC) is not used for forecasting the state of the junction in the next minutes. This approach could be incorporated to estimate the traffic condition in the close future and better optimise the control policy.

The approximations and errors induced by the listed assumptions and limitations, and the consequences in relaxing those are discussed in the rest of the paper.

\subsection{Control strategy analytical formulation}

The equations governing the CoopMA system need to define in a quantitative way the following aspects.

\section{A.1 the maximum size of the gap $G$}

\section{A.2 the traffic light cycle and phases}

\section{A.3 the maximum on-ramp flow}

\section{A.4 the time and time needed to compact the vehicles}

The evaluation of these aspects can give an insight on the practicality of the CoopMA system. For example, if the cooperative vehicle must keep a slow speed for an excessive long stretch of motorway, e.g. several kilometres upstream of the merging section, the entire system can be considered impractical, because it is not reasonable to suppose that a driver will proceed at a low speed for a long time. Therefore, the control strategy formulation here developed is also useful to investigate the practicality of the CoopMA. The conclusions on the practicality of the system will be based on what can be considered reasonable, without fixing qualitative criteria.

To evaluate these aspects, we use a combination of macroscopic traffic flow theory and microscopic considerations. The fundamental diagram and its properties have been used to determine the size of the gap $G$. Meanwhile, the spatio-temporal diagram and shock wave theory have been used to define the time and space required to create the gap, i.e. the spatio-temporal evolution of the fronts between the traffic state $A, C$ and $O$. Finally, being the CoopMA an Active Traffic Management system controlling individual vehicles, the macroscopic theory has been combined with microscopic considerations on vehicle trajectories, and characteristics as well as driver behaviour.

The fundamental diagram describes the relationships among speed $v$, flow $q$ and density $k$. These variables are used to describe traffic flow at macroscopic level.Every traffic state, denoted by $\phi$, is defined univocally by its speed $v_{\phi}(\mathrm{km} / \mathrm{h})$, density $k_{\phi}(\mathrm{veh} / \mathrm{km})$ and flow $q_{\phi}(\mathrm{veh} / \mathrm{h})$.

The spatio-temporal diagram is used to investigate the evolution of these variables in space and time. This tool is necessary because traffic phenomena are usually not stationary, and develop in space and time in a dynamic way, in particular in congested situations.

The fundamental diagram of traffic flow and the spatio-temporal diagram are deeply related. The theory that links the two is known as shock waves theory or kinematic wave model (Lighthill and Whitham, 1955). In the fundamental diagram, Figure 4(a), the slope of the line tangent to the curve at any traffic state $\phi$ defines the speed at which that state propagates in the spatio-temporal plane, thin 


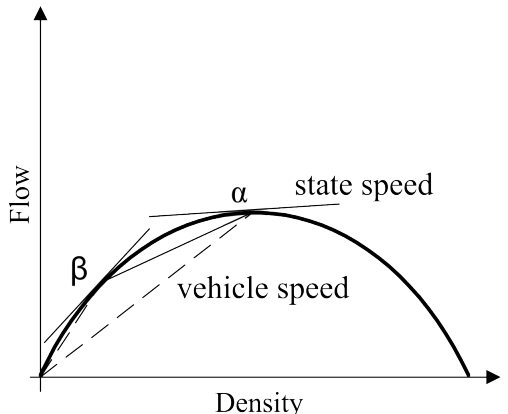

(a)

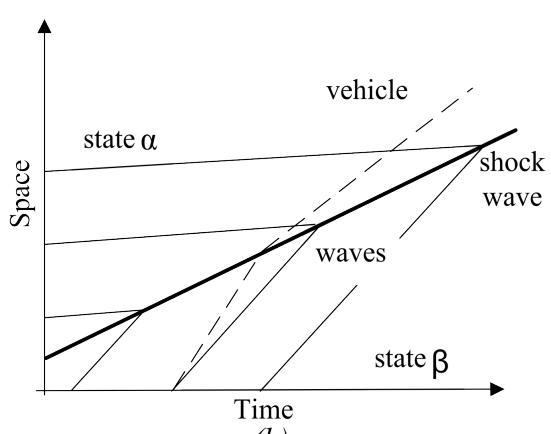

(b)

Figure 4: Graphical representation of the shock wave theory. (a) shows the fundamental diagram with vehicle speed, state speed and front speed. (b) presents the spatio-temporal diagram with the front propagation. Adapted from Lighthill and Whitham (1955).

solid lines in Figure 4(b). This speed is not the speed of the vehicles, but the speed of the state $\phi$. The vehicle speed is defined by the slope of the line connecting the traffic state to the origin, dashed lines in Figure 4(a). In case two traffic states are present in two different motorway sections, e.g. traffic state $\alpha$ downstream of an on-ramp and traffic state $\beta$ upstream, this traffic flow theory can also be used to identify the speed of the front between the traffic states. The slope of the line connecting the two states represents the speed at which the front propagates in the space-time diagram, the bold solid line in Figure $4(b)$. In this case, the speed is positive and so the front propagates downstream. Fronts between states that have lower speed downstream are also referred to as shock waves.

In the following, we present the considerations used to derive the equations defining the aspects A1-A4 introduced at the beginning of this section.

The idea of the Cooperative MA illustrated with the spatio-temporal diagram in Figure 1(c) can be explained using the fundamental diagram of traffic flow. Assuming that the actual traffic is in state $A$, using the fundamental diagram it is possible to know its speed $v_{A}$, density $k_{A}$ and flow $q_{A}$, identified by point $A$ in Figure 5. The vehicles in state $A$ travel with a headway, the time between the passing of the front of two successive vehicles over the same point, equal to $h_{A}$ (seconds), and the spacing, the distance between the fronts of two successive vehicles, equal to $s_{A}$ (metres), as shown in Figure 1(a). If the cooperative vehicle slows down to speed $v_{C}$, after a certain amount of time, the vehicles immediately upstream will travel at a higher density $k_{C}$, state $C$ in Figure 5 . The spacing between the vehicles is reduced, Figure 1(b); therefore, if a consecutive cooperative vehicle is set, a gap $G$ (seconds), the time between the passing of the rear of the leading vehicle and the front of the following vehicle over the same point, is artificially created between the last vehicle in the platoon and the next cooperative vehicle, as shown in Figure 1(b). For clarity, the normal gap between the vehicles is identified by the symbol $g_{\phi}$ (seconds), indicating the gap for the traffic state $\phi$, derivable from Eq. (1)

$$
g_{\phi}=q_{\phi}^{-1}-L v_{\phi}^{-1}
$$

where $L$ is the length of a vehicle. Instead, the gap created in front of the cooperative vehicle is identified by the symbol $G$ (seconds).

In summary, reducing the speed of the cooperative vehicle, it is possible to modify the traffic flow from state $A$ (actual state) to state $C$ (cooperative state), with the addition of an empty area, state $O$ (origin state). It is immediately clear that the size, in time or space, of this gap $G$ is a function of state $A$, state $C$ and the number of vehicles between two consecutive cooperative vehicles, including the cooperative one, defined as the platoon size $n_{\mathrm{p}}$. Thus, state $A$ can be defined as an external input, and $v_{C}$ and $n_{\mathrm{p}}$ as CoopMA design variables.

To have an analytical description of the conceptual fundamental diagram presented in Figure 5, a model has been fitted to real data provided by the MIDAS system. MIDAS is a distributed network of detector inductive loop that covers about a third of English motorway, spaced at 500 metres intervals (DfT, 2011; HA, 2007). This approach does not lead to a loss of generality because, the chosen bottleneck has typical characteristics observed in literature. Figure 6 shows MIDAS detector loop data of an English motorway junction that behaves as an active bottleneck. As will be clarified subsequently, for the CoopMA formulation, it is necessary that the model of the fundamental diagram represents accurately only the free-flow section. This is because, an assumption of the system is that the cooperative vehicle 


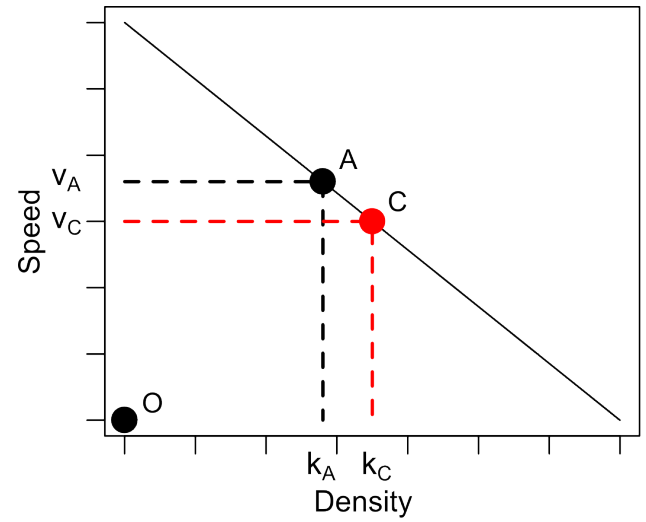

(a)

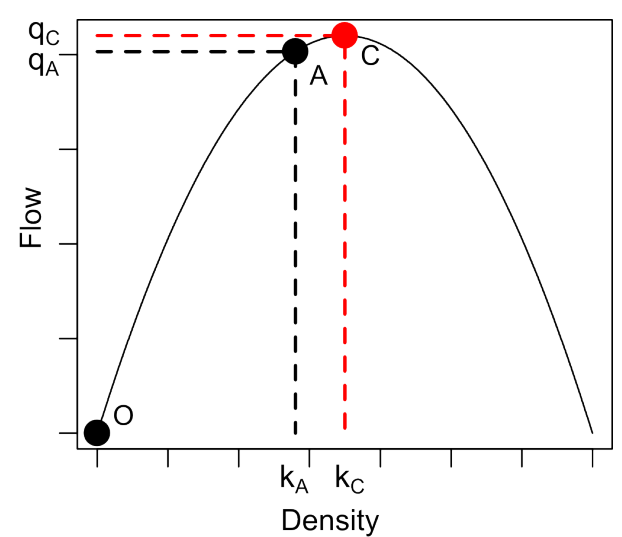

(b)

Figure 5: CoopMA traffic states. (a) speed-density and (b) flow-density diagrams with the representation of the different traffic state created by the cooperative vehicle. Adapted from (Scarinci et al., 2015).

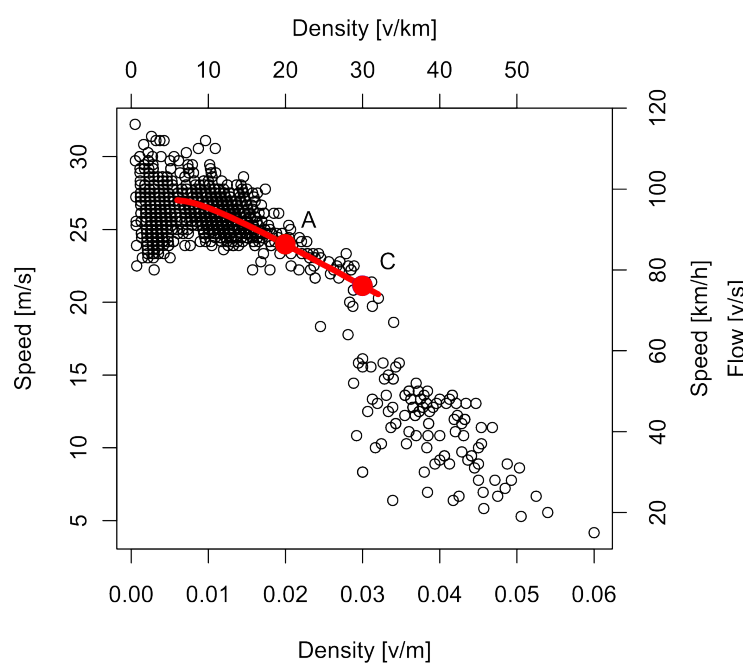

(a)

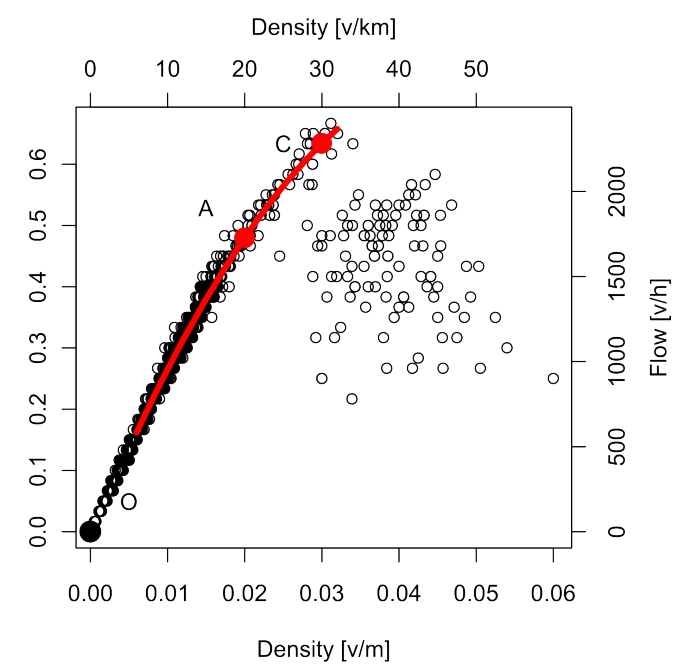

(b)

Figure 6: Detector loop data (MIDAS English motorway M6-J19) and fitted model with the representation of the actual $A$ and cooperative $C$ traffic states. (a) speed-density and (b) flow-density plane. 
speed $v_{C}$ is always higher than the critical speed. Therefore, the system operates only in the freeflow state, so there is no need to estimate the congested section. The boundary between the free and congested states, clearly visible in the real data in Figure 6, is delimited by the critical speed $v^{*}$. This speed, according to traffic flow theory, is the slope of the line connecting the points of maximum flow to the origin in the flow-density diagram, equal to $70 \mathrm{~km} / \mathrm{h}$ analysing the MIDAS data in Figure 6 . Having defined the boundary between free-flow and congested-flow, a model for the free section can be estimated. A parabola has been fitted using a standard least square regression approach in the plane $q-k, q(k)=a k^{2}+b k+c$. The results are reported in Figure 6 and Eq. (2), where the coefficient values are for flow in veh $/ \mathrm{h}$ and density in veh $/ \mathrm{km}$.

$$
q(k)=-1.04 k^{2}+109 k-34.1 .
$$

We define as $\Delta v$ the speed difference between the actual speed of the traffic $v_{A}$ (measured), and the speed to which the cooperative vehicle slows down $v_{C}$ (design variable):

$$
\Delta v=v_{A}-v_{C} .
$$

This reduction in speed defines how strong the disruption caused to the traffic flow is. The speed $v_{C}$ of the cooperative vehicle should be chosen to lie in the range from $v_{A}$ to the speed that maximises flow, i.e. the critical speed $v^{*}$. This is because $v_{C}$ should be slow enough to create a gap $G$ in a reasonable amount of time, but high enough to avoid flow break-down. Macroscopic traffic flow theory provides a possible methodology to identify the minimum speed for the cooperative vehicle. If, for example, we suppose that the fundamental diagram can be described by the Greenshields (1935) model, then if $v_{C}$ is greater than $v^{*}$, the associated wave speeds will all be positive. As a consequence, disruptions do not propagate upstream and lead to break-down. Therefore, to maintain the state $C$ in the free-flow section, we define the maximum value of $v_{C}$ as

$$
v_{C}=\max \left(v_{A}-\Delta v, v^{*}\right) .
$$

This means that, if traffic state $A$ is close to the critical state, i.e. $v_{A}$ is close to $v^{*}$, the speed difference between the cooperative vehicle $v_{C}$ and the other vehicles $v_{A}$ decreases until 0 . So, $\Delta v$ is a CoopMA design variable, defining the maximum desired decrease in speed, and it should satisfy Eq. (4) for stability reasons.

This macroscopic consideration on the system stability cannot be considered completely accurate, due to the stochasticity of capacity (Prigogine and Herman, 1971) and the critical amplitude of the perturbations (Ranjitkar et al., 2003). However, the algorithm can prevent shock waves by appropriately slowing down the vehicles, such that the resulting traffic state is in free flow. Free flow means that the characteristics propagate forward and no shock waves will be created. This approach is widely used for defining ATM control strategies (Kerner and Rehborn, 1997; Hegyi et al., 2008) and has been tested and verified in practice with even more aggressive vehicle decelerations (Hegyi and Hoogendoorn, 2010). In the current approach, the prevention of the shock waves is guaranteed by an appropriate choice of the speed of the cooperative vehicle $v_{C}$, which should be sufficiently high to ensure free flow. These theoretical considerations have been verified using microscopic simulation. Section 5 reports the main results showing that the deceleration of the cooperative vehicles using the aforementioned approach does not create shock waves. A complete discussion on these aspects can be found in Scarinci et al. (2015). Another consideration should be made on the maximum value of $\Delta v$. It is not possible to set a large difference between the speed $v_{A}$ and $v_{C}$, because high speed difference between vehicles could lead to unsafe situations and collisions. From the theory on speed limit control, motorway operators require increments or decrements in speed between two consecutive variable message signs (VMS) between 10 $\mathrm{km} / \mathrm{h}$ and $30 \mathrm{~km} / \mathrm{h}$ (Hegyi, 2004; Hegyi and Hoogendoorn, 2010). We use the same constraints on $\Delta v$.

Using the fitted model of the fundamental diagram and the previous equations, it is possible to define the gap $G$ (seconds) achievable for the different traffic conditions following straightforward consideration. As shown by Figure 5, once the CoopMA is applied, the flow in state $A$, is different from the flows in states $C$ and $O, q_{A} \neq q_{C} \neq q_{O}$, however, the average flow remains the same. This is because, the Cooperative MA system, maintaining $v_{C}$ always greater than $v^{*}$, does not limit the total flow on the motorway, but it splits the traffic flow in state $A$ to the traffic flow in states $C$ and $O$. Therefore the flow $q_{A}$ is equal to the average flow of states $C$ and $O, q_{A}=\bar{q}_{C O}$, where the state $C O$ is the union of states $C$ and $O$, i.e. the platoon of vehicles plus the gap created, and the bar indicates the average. The same consideration can be done for the headway $h_{\phi}=1 / q_{\phi}$ (seconds). Therefore $h_{A}=\bar{h}_{C O}$, but $h_{A} \neq h_{C} \neq h_{O}$. It is now possible to define the total spacing between two cooperative vehicles as

$$
s_{C}^{\text {tot }}=s_{C} \cdot n_{\mathrm{p}}+G^{\mathrm{s}}
$$


where $s_{C}$ (metres) is the spacing between two consecutive vehicles at traffic state $C$, and $n_{\mathrm{p}}$ is the platoon size. $G^{\mathrm{s}}$ is the quantity of interest, i.e. the gap in space (clearance) created by the speed reduction of the cooperative vehicle. Figure $1(b)$ can be used to visualize this equation. Eq. (5) defines $G^{\mathrm{s}}$ once the vehicles have finished the transition from state $A$ to state $C$, i.e. they have completed the compacting process, and so the gap is maximum and henceforth remains constant. It is now possible to derive $G^{\mathrm{s}}$ from Eq. (5), and remembering $s_{\phi}=h_{\phi} \cdot v_{\phi}$, it is obtained

$$
G^{\mathrm{s}}=\left(n_{\mathrm{p}} \cdot v_{C}\right) \cdot\left(h_{A}-h_{C}\right) .
$$

Being $v_{C} \leq v_{A}$ but always greater than $v^{*}, q_{C} \geq q_{A}$ and so $h_{C} \leq h_{A}$. These inequalities applied to Eq. (6) confirm that $G^{\mathrm{s}} \geq 0$. This means that the reduction in speed of the cooperative vehicle creates a usable gap, as expected. The size of the gap is proportionate to the difference in headway between state $A$ and $C$, as shown by Eq. (6). Finally, to define the correct $G^{\mathrm{s}}$, the $g_{C}^{\mathrm{s}}$, i.e. the gap in space (clearance) between two consecutive vehicles during the traffic state $C$, of the last vehicle in the platoon must be added because this gap is considered useful space for merging. Therefore, the final equation for the gap in space, defined as $G^{\mathrm{s}^{\prime}}$ is

$$
G^{\mathrm{s}^{\prime}}=G^{\mathrm{s}}+g_{C}^{\mathrm{s}}
$$

So far it has been convenient to define the gap $G$ as clearance, i.e. focusing on the space, to avoid the complication of visualizing the traffic state speed too. The gap in time created in front of the cooperative vehicle can be calculated using

$$
G=G^{\mathrm{s}^{\prime}} / v_{C}
$$

Here, $v_{C}$ is used because the speed of the two ends of the gap is equal to the cooperative vehicle speed once the vehicles are completely compacted, as shown in Figure 1(c).

Having defined $G$, the maximum number of on-ramp vehicles $n_{\mathrm{o}}$ able to merge in this gap must be determined. For the definition of the control strategy, a simple hypothesis is made on the merging behaviour, i.e. all vehicles need the same amount of time to merge, denoted by $g_{\mathrm{m}}$. Therefore, the number of on-ramp vehicles able to merge $n_{\mathrm{o}}$ is given by the ratio between the gap created $G$ and the average gap that a vehicle needs for merging $g_{\mathrm{m}}$. Because only an integer number of vehicles can merge, $n_{\mathrm{o}}$ is calculated by truncating the number of vehicles to the greatest integer that does not exceed the time gap for merging. Thus:

$$
n_{\mathrm{o}}=\operatorname{Int}\left(G / g_{\mathrm{m}}\right) \text {. }
$$

Calculating $n_{\mathrm{o}}$ using Eq. (9) can be considerate a conservative approach. When a platoon of vehicles merges, the total gap necessary is less than the sum of the gap necessary for each individual vehicles $g_{\mathrm{m}}$. Furthermore, the total gap can be also reduced with the presence of intelligent vehicles able to travel in a platoon formation. Anyway, this conservative approach has been chosen to ensure a gap theoretically large enough for $n_{\mathrm{o}}$ vehicles.

Finally, knowing the number of vehicles merging at each traffic light cycle, Eq. (9), the maximum hourly on-ramp flow is then calculated as

$$
q_{\mathrm{o}}^{\max }=n_{\mathrm{o}} / C_{\mathrm{c}}
$$

where, $C_{\mathrm{c}}$ (seconds) is the cycle of the cooperation, a direct consequence of the number of vehicles in the platoon and their headway at state $A$ :

$$
C_{\mathrm{c}}=h_{A} \cdot n_{\mathrm{p}}
$$

Different considerations are used to determine the time and space required to create the gap $G$. These quantities are of fundamental importance, because they define when and where to send a message to the cooperative vehicle requesting the deceleration. In order to define the time and space needed, it is possible to use shock wave theory (Lighthill and Whitham, 1955). In the specific case of CoopMA these are represented in Figure 7. Figure $7(a)$ has been used to define the traffic states $A, C$ and $O$, and Figure $7(b)$ describes the evolution of the fronts of these states in space and time. According to the shock wave theory, the speed of the front between state $A$ and $C, v_{A C}$, the dashed line in Figure $7(b)$, is equal to the slope of the line connecting the states on the flow-density diagram, the dashed line connecting $A$ and $C$ in Figure $7(a)$.

Combining the front trajectory, i.e. the speed of the front $v_{A C}$, with the trajectory of individual vehicles composing a platoon, the solid lines in $7(b)$, it is possible to determine the time and space that the vehicles in the platoon will need to compact and to create the gap, i.e. change from state $A$ to state $C$ and $O$. Therefore, using linear algebra in the spatio-temporal diagram plane, it is possible to define 


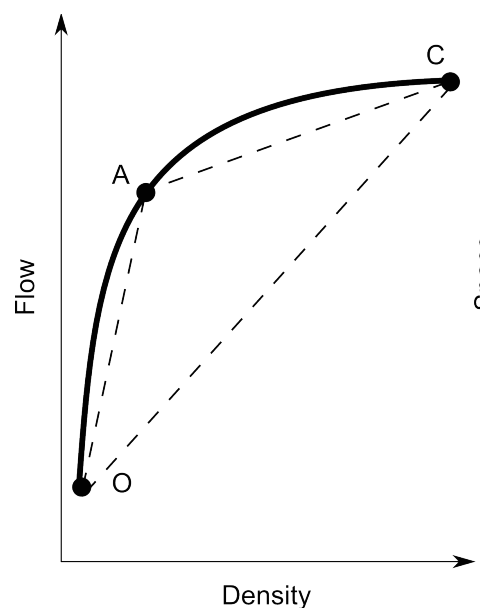

(a)

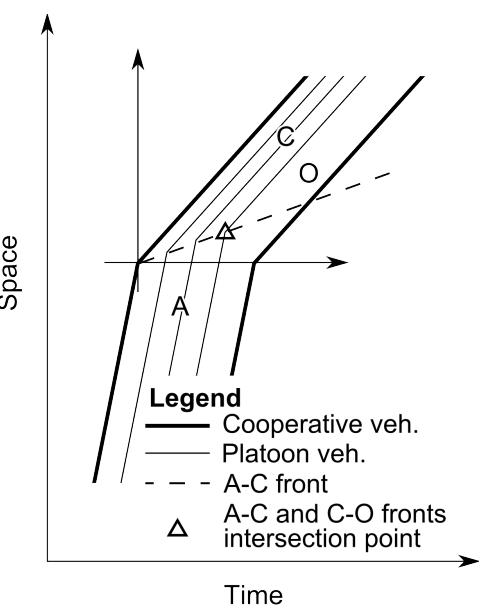

(b)

Figure 7: Trajectories of the CoopMA traffic state fronts. (a) conceptual illustration of the flow-density fundamental diagram and representation of the front propagation speed. (b) conceptual spatio-temporal diagram of the vehicles trajectory during the different traffic states with representation of the fronts between the states $A, C$ and $O$.

the time $t_{A C}$ necessary for complete the compacting process. This point is represented by a triangle in Figure 7(b), and it is identified by the intersection between the trajectory of the last vehicle in the platoon and the front between state $A$ and $C$. Fixing the origin of the coordinate system at the point where the cooperation starts, the equation of the trajectory of the last vehicle in the platoon is given by

$$
x_{\mathrm{n}}=v_{A} \cdot t-s_{A} \cdot\left(n_{\mathrm{p}}-1\right),
$$

where $x_{\mathrm{n}}$ defines the position of vehicle $n, t$ defines the time and $s_{A}$ is the spacing between vehicles at state $A$. The equation of the front propagation between state $A$ and $C$ is given by

$$
x_{A C}=v_{A C} \cdot t,
$$

where $v_{A C}$ is defined by the model of the fundamental diagram

$$
v_{A C}=\frac{q_{C}-q_{A}}{k_{C}-k_{A}} .
$$

Equaling Eq. (12) and Eq. (13) to identify the intersection between the lines, i.e. the triangle in figure, the time $t_{A C}$ necessary for the change of state is given by the following equation:

$$
t_{A C}=\frac{-s_{A} \cdot\left(n_{\mathrm{p}}-1\right)}{v_{A C}-v_{A}} .
$$

Finally, to estimate the total cooperation time $t_{C}$, in case of on-board display technology, the driver reaction time $t_{\mathrm{r}}$ to the displayed message should be added to the time necessary to change traffic states

$$
t_{C}=t_{\mathrm{r}}+t_{A C} .
$$

At this point, using simple kinematic equations, it is possible to define the cooperation space $x_{C}$, i.e. the point at which the cooperative vehicle should receive the message of decreasing the speed. This distance is given by

$$
x_{C}=x_{\mathrm{r}}+x_{A C},
$$

where $x_{\mathrm{r}}$ is the space which the cooperative vehicle covered before reacting at the message

$$
x_{\mathrm{r}}=v_{A} \cdot t_{\mathrm{r}},
$$

and $x_{A C}$ is the space necessary for the last vehicle in the platoon to compact

$$
x_{A C}=v_{A C} \cdot t_{A C} .
$$


In Eq. (18) the used speed is $v_{A}$ because the driver has not yet reacted to the instruction of decreasing its speed, and in Eq. (19) the speed is not $v_{C}$, i.e. the vehicle speed when the driver has completed the deceleration, but the one of the front between state $A$ and $C$, i.e. $v_{A C}$, because it is necessary to define when the downstream front of the gap $G$ will reach the merging location, and not when the cooperative vehicle will reach it, as visible in Figure $7(b)$.

It is possible to extend the equations for $t_{C}$ and $x_{C}$ considering that the cooperative vehicle does not change speed instantaneously, but it follows a constant deceleration $d$ from $v_{A}$ to $v_{C}$. It has been estimated that the driver's reaction to a reduction in the speed limit is to release the throttle without active braking, creating an average deceleration in the order of 0.3 to $0.5 \mathrm{~m} / \mathrm{s}^{2}$ (Netten et al., 2011; Daamen et al., 2011). We use kinematic equations to estimate the effect of this smooth transition from speed $v_{A}$ to speed $v_{C}$. The constant deceleration results in a small translation downstream of the individual vehicle trajectories shown in Figure $7(b)$. This translation effect does not influence $t_{A C}$ significantly because $\Delta v$ is limited by the safety consideration discussed earlier, and the extra time needed will be incorporated by the use of a safety factor that will be introduced in the next section. For this reason, we do not consider this effect in our macroscopic control strategy. However, we note that acceleration and jerk play an important role. Control strategies minimizing accelerations and decelerations are able to reduce emissions, and improve passenger's comfort and safety (Ntousakis et al., 2016).

Having defined the traffic signal cycle in Eq. (11), the final quantities that must be defined to have a complete control policy are the start and duration of the green phase. The start of the green phase is triggered by the position of the cooperative vehicle communicated to the infrastructure. The green phase will begin when the predicted arrival at the merging location of the gap $G$ will match the predicted arrival of the on-ramp vehicles released by the traffic light. This means that each cooperative vehicle triggers the green phase for the vehicles that will merge in front of it. The relative arrival between the on-ramp vehicles and the cooperative vehicles can be estimated again using kinematic equations. The on-ramp vehicles are assumed to have a uniformly accelerated motion from zero speed when released by the traffic light, instead the main carriageway vehicles will follow the trajectories described earlier. Based on this consideration, it is possible to define the start time of the green phase knowing the average vehicle acceleration and the junction geometry. From classic kinematic equations, $v_{t}=v_{o}+a t$ and $x_{t}=x_{o}+v_{o} t+1 / 2 a t^{2}$, the travel time of the on-ramp vehicles from the stop line to the merging location is calculated, and the traffic light green phase started accordingly. The green phase, i.e. the number of vehicles released in each traffic light cycle, is instead proportional to the size of the gap $G$. The duration of the green phase is calculated so that the number of vehicles released is the maximum able to merge in the gap $G$, defined by Eq. (9). Traditional RM installations calculate that a vehicle needs a fixed time $c_{g}^{n}$ of 2 second to cross the stopping line during the green phase (Papageorgiou and Papamichail, 2008), therefore the green phase $C_{g}$ (second) is equal to

$$
C_{g}=n_{\mathrm{o}} \cdot c_{g}^{n} .
$$

Assuming the absence of an amber phase, all the traffic light phases are defined, being the red one $C_{r}$ just the difference between the cycle $C_{c}$, Eq. (11), and the green phase $C_{g}$

$$
C_{r}=C_{c}-C_{g} .
$$

Therefore, the green phase and the cycle length are control variables, and the red phase is derived. Often minimum cycle, red and green time are imposed by the operators, and these should be used as a constraint in defining the traffic light phases.

The present set of equations, Eq. (2)-(21), composes the complete Cooperative Merging Assistant control strategy analytical formulation, which will be implemented in Section 5 for the evaluation of the system traffic performance.

\subsection{Results and discussion}

The analytical formulation derived in the previous section can be now used to define the CoopMA aspects (A1-A4) presented at the beginning of the previous section. The equations are a function of the external input not controlled by the system, i.e. traffic state $A$, the design variables $\Delta v$ and $n_{\mathrm{p}}$, and the other parameters presented, e.g. fundamental diagram model Eq. (2), $v^{*}$ critical speed, $g_{\mathrm{m}}$ minimum gap for merging, $t_{\mathrm{r}}$ driver's reaction time, $a$ vehicle acceleration. In order to visualize in two dimensions the results of the equations, it is convenient to fix some of these parameters and evaluate the trends for few 
Table 1: Parameter values used for the graphical representation of the CoopMA analytical formulation

\begin{tabular}{lcll} 
Parameter & Value & Unit & Source \\
\hline$g_{\mathrm{m}}$ & 3.0 & $\mathrm{sec}$ & (Daamen et al., 2010) \\
$\Delta v$ & 10.0 & $\mathrm{~km} / \mathrm{h}$ & (Hegyi, 2004) \\
$d$ & -0.5 & $\mathrm{~m} / \mathrm{s}^{2}$ & (Daamen et al., 2011) \\
$t_{\mathrm{r}}$ & 1.5 & $\mathrm{sec}$ & (Netten et al., 2011) \\
$v^{*}$ & 70.0 & $\mathrm{~km} / \mathrm{h}$ & MIDAS data \\
$a$ & 3.0 & $\mathrm{~m} / \mathrm{s}^{2}$ & (FHA, 2010) \\
Traffic light & minimum phase & (Papageorgiou and Papamichail, 2008) \\
$\quad$ red & 2.0 & $\mathrm{sec}$ & \\
$\quad$ green & 2.0 & $\mathrm{sec}$ & \\
$c_{g}^{n}$ & 2.0 & $\mathrm{sec}$ & \\
\hline
\end{tabular}

variables. Being among the most important aspects, the CoopMA aspects have been answered varying the traffic state $A$ and the platoon size $n_{\mathrm{p}}$.

Figure 8 shows the results of the analytical formulation for the parameter values reported in Table 1. The abscissa in all the sub-figures represents the traffic flow on the motorway near-side lane, i.e. the $A$ state. The ordinate represents one different result for each figure, in order: (a) the gap $G$ created in front of the cooperative vehicle, $(b)$ the cooperative vehicle cycle $C_{c},(c)$ and $(d)$ the maximum on-ramp flow $q_{\mathrm{o}}^{\max }$ with and without the constraint of the integer number of vehicles able to merge respectively, $(e)$ the time $t_{C}$ needed for generating the gap and $(f)$ the relative space $x_{C}$. All these indexes are presented for state $A$ from $1000 \mathrm{veh} / \mathrm{h}$ (representative of uncongested situation) to $2250 \mathrm{veh} / \mathrm{h}$ (close to capacity as estimated by MIDAS data, Figure 6), and platoon size $n_{\mathrm{p}}$ from 2 (minimum size) to 20 vehicles (choice based on practicality consideration).

Figure 8(a) shows the size of the gap $G$, Eq. (5)-(8). According to the expectations, $G$ decreases with the increase of the traffic flow on the main carriageway because fewer empty spaces are left for rearranging the vehicles. Larger platoons on the main carriageway are able to create bigger gaps, but, as shown by Figure $8(b)$, with smaller frequency. It is also clearly visible the change in slope due to the limitation on the maximum difference in speed $\Delta v$, that limits $v_{C}$ in being always higher than the critical speed, Eq. (4). The dotted horizontal lines represent the average gap $g_{\mathrm{m}}$ that a vehicle needs to merge. Therefore the number of dotted lines under the solid line represents the number of vehicles able to merge in that gap. Figure $8(b)$ shows the cooperation cycle $C_{c}$, i.e. how often it is possible to set a cooperative vehicle, Eq. (11), for the different platoon sizes. This time is proportional to the headway of the vehicles on the main carriageway as explained during the definition of the analytical formulation. Logically, to form bigger platoons more time is needed. Figure 8(c) describes the maximum on-ramp flow $q_{\mathrm{o}}^{\max }$ achievable given the combination of the gap created and the cooperation cycle, Eq. (9)-(10). Because only an integer number of vehicles can merge, the maximum flow is represented by a broken line, and then the resulting flows are also discontinuous. The figure shows that different platoon sizes provide the maximum flow for different traffic states $A$. This is particularly relevant for small platoons, because a slight change in the gap $G$ could lead to a significant reduction in the number of vehicles able to merge, for example, from 2 to 1 vehicles per cycle, or even from 1 to 0 , meaning that the gap created is not enough for any vehicle to merge. This sharp loss in merging capacity is represented by the vertical lines in Figure 8(c). For larger platoon size, the loss in capacity is less relevant, and, as visible in figure, the broken lines converge toward the continuous line. For a better understanding of the trends, Figure 8(d) represents the same maximum on-ramp flow without the constraint that only integer numbers of vehicles can merge. As for the previous figure, a significant change in capacity is observable increasing the size of small platoons, e.g. from 2 to 3 vehicles. While, for large platoons, e.g. 20 vehicles, an increase brings only a marginal change, as visible from the lines converging to a lower limit. So, it is possible to conclude that, with the hypothesized merging behaviour, smaller and more frequent gaps are more efficient than bigger and less frequent ones. This is because the naturally present intra-vehicles clearance $g_{C}^{\mathrm{s}}$ of the last vehicle in the platoon is used as available space for merging, and, as shown by Eq. (7), one $g_{C}^{\mathrm{s}}$ is added for each platoon independently of $n_{\mathrm{p}}$. This consideration holds only assuming that platoon of vehicles required the same amount of gap than individual vehicles. The consequences of relaxing this strong assumption are left for further work. Finally, Figure $8(e)$ and $(f)$ indicate when $t_{C}$ 


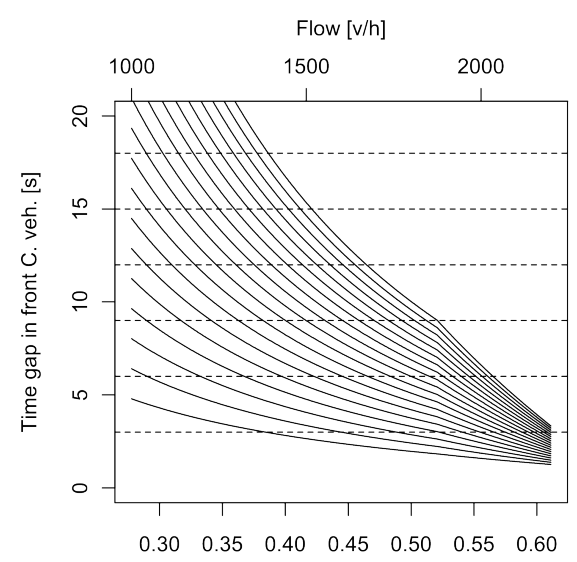

(a) Flow $[v / s]$

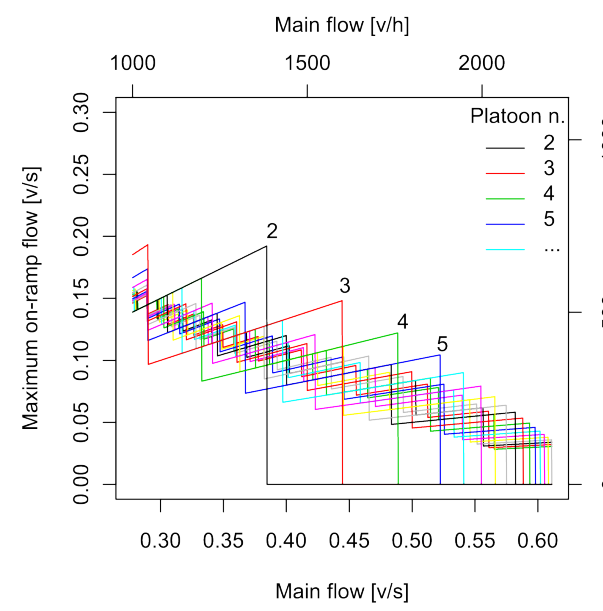

(c) Flow $[\mathrm{v} / \mathrm{h}]$

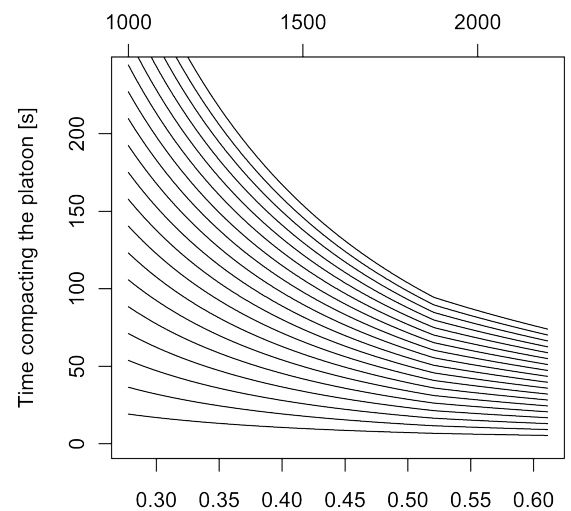

(e) Flow $[v / s]$

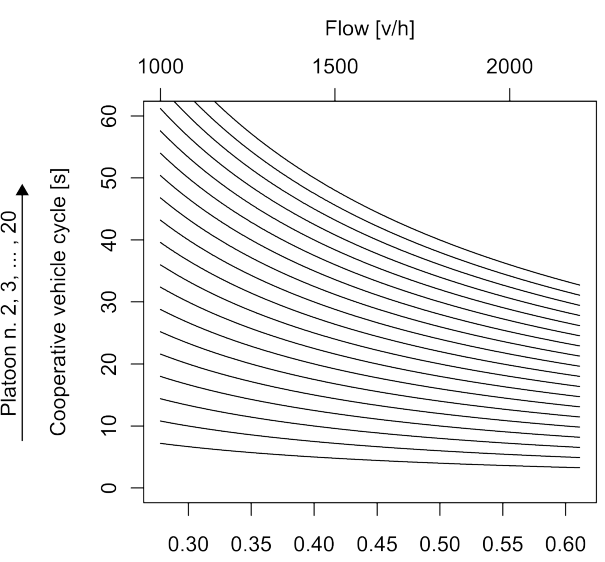

(b) Flow [v/s]

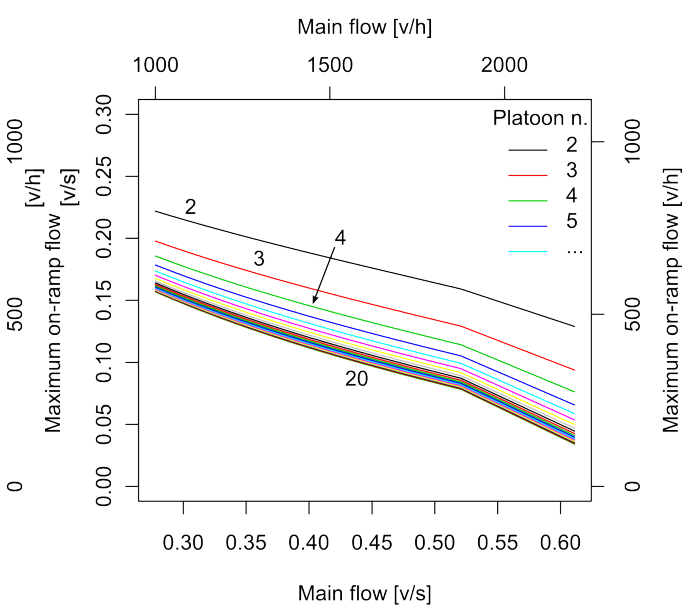

(d) Flow $[\mathrm{v} / \mathrm{h}]$

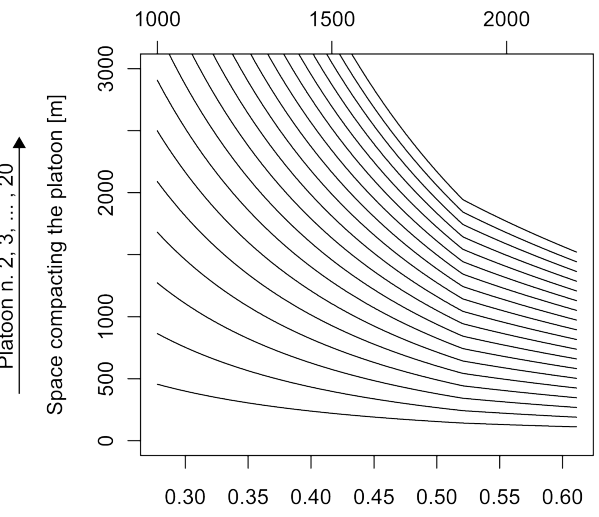

(f) Flow $[\mathrm{v} / \mathrm{s}]$

Figure 8: CoopMA formulation main results. (a) gap $G$ created in front of the cooperative vehicle, (b) cooperative vehicle cycle $C_{c}$, (c) maximum on-ramp flow $q_{\mathrm{o}}^{\max }$ with the constraint of integer number of vehicles able to merge and $(d)$ without this constraint, $(e)$ time $t_{C}$ needed to create the gap and $(f)$ the relative space $x_{C}$. 
and where $x_{C}$ the cooperation should start in order to provide a gap for the on-ramp merging vehicles, respectively Eq. (15)-(16) and Eq. (17). As expected, the time and space for the cooperation increase significantly with the increase of the platoon number, and with the reduction of main carriageway flow. This is because vehicles have a greater distance between them, so they need more time to compact.

In summary, Figure 8 gives an analytical answer to the CoopMA aspects, and is also useful to discuss the practicality of the system. For example, supposing that the traffic flow on the near-side lane of the main carriageway is $1,500 \mathrm{veh} / \mathrm{h}$, the chart on Figure 8(a) shows that with the generation of a seven vehicle platoon, a gap of 6 seconds can be created every 16 seconds, Figure $8(b)$. This gap will provide a maximum on-ramp flow of $450 \mathrm{veh} / \mathrm{h}$, Figure 8(c), flow similar to traditional RM (Papageorgiou and Kotsialos, 2002). The in-car message to the cooperative vehicle with the information of the speed to maintain should be sent 60 seconds before the gap reaches the merging location, i.e. about 1100 metres upstream. Given this example and the trends visible from the analytical results, it is possible to assert that the size of the gap achievable and the time-space needed for its creation, i.e. the requested cooperation, are reasonable and compatible with a real deployment of the system. Therefore, the system could be considered practical.

\section{Control strategy validation}

We here validate the analytical formulation presented in the previous section. The same analytical results are re-created starting from a different approach, based on simulation rather than theory.

Section 4.1 describes the simulation scenario and the procedure adopted to calculate the indexes. The simulation results, a qualitative discussion of their trends and a more formal comparison between theoretical and simulation indexes are presented in Section 4.2.

\subsection{Simulation scenario and index calculation}

The same results visualized in Figure 8 calculated using the analytical formulation are here estimated starting from the behaviour of individual vehicles generated with a microscopic simulation model.

The simulation scenario has been designed to recreate the compacting effect with consequent platoon formation caused by the decrease in speed of a cooperative vehicle. Because the analytical formulation is based on macroscopic considerations that do not consider inter-vehicle variability, the simulation has been based on the same assumptions. Therefore vehicles with identical characteristics (including desired speed, acceleration, car following behaviour) are generated with a constant headway.

The combination of the programming language MATLAB - version R 2012b (MathWorks, 2013) and the microscopic traffic model VISSIM - version 5.40 (PTV, 2013) has been adopted for the calculations used to investigate the validity of the CoopMA system. MATLAB defines the flow and platoon size, and generates vehicles on the infrastructure as well as modifying the desired speed of the cooperative vehicles when they enter the transition section. VISSIM is used to move the vehicles along the motorway using its internal car-following model and to record raw data, e.g. vehicle position and speed at each time step. VISSIM uses a psycho-physical car following model based on Wiedemann (1974) and a lanechanging/merging model based on consecutive logical actions incorporating courtesy lane-changing and courtesy yielding behavior. This tool has been used in recent evaluations of cooperative merging control strategies (Park et al., 2011; Marinescu et al., 2012). Finally, the recorded data are analysed in MATLAB in order to obtain the indexes. The exchange of information between VISSIM and MATLAB has been achieved using the COM interface, a protocol enabling access to VISSIM data allowing the software to work as an Automation Server.

As for the theoretical results, the simulation indexes have been evaluated for a range of main carriageway flows from $1000 \mathrm{veh} / \mathrm{h}$ to $2250 \mathrm{veh} / \mathrm{h}$, and different platoon sizes, from 2 to 20 . Table 2 summarises the parameters used. Eleven different flows have been simulated: $1000 \mathrm{veh} / \mathrm{h}, 1059 \mathrm{veh} / \mathrm{h}, 1125 \mathrm{veh} / \mathrm{h}$, $1200 \mathrm{veh} / \mathrm{h}, 1286 \mathrm{veh} / \mathrm{h}, 1385 \mathrm{veh} / \mathrm{h}, 1500 \mathrm{veh} / \mathrm{h}, 1636 \mathrm{veh} / \mathrm{h}, 1800 \mathrm{veh} / \mathrm{h}, 2000 \mathrm{veh} / \mathrm{h}, 2250 \mathrm{veh} / \mathrm{h}$. These flows are not round numbers due to the simulation internal resolution, i.e. time is discrete and not continuous. Therefore, due to the necessity to keep a constant headway, vehicles can be generated only at specific time steps. Each platoon size is evaluated for each different flow giving a total of 209 simulations (11 flows times 19 platoon sizes). The simulated time horizon is 30 minutes, chosen to be sufficiently long that at least 10 platoons complete the formation process, giving the possibility to average the indexes over multiple events. The demand is represented by two types of vehicles, cooperative and normal, with identical characteristics, but during the simulation, the desired speed of the cooperative vehicles is reduced while no modifications are made to the normal ones. 
Table 2: Parameter values used for the CoopMA simulation

\begin{tabular}{lllll} 
Parameter & Min & Max & Step & Unit \\
\hline Main carrigage flow & 1000 & 2250 & $\sim 50$ & veh/h \\
Platoon size & 2 & 20 & 1 & veh \\
Simulated time & 30 & - & - & min \\
Total simulations & 209 & - & - & - \\
\hline
\end{tabular}

The infrastructure is composed of a stretch of motorway of $11 \mathrm{~km}$, single lane divided it into three logical sections: a generation section from $\mathrm{km} 0$ to $\mathrm{km} \mathrm{1}$, a transition section from $\mathrm{km} 1$ to $\mathrm{km} 10$ and a control section from $\mathrm{km} 10$ to $\mathrm{km} 11$. The vehicles are generated at $\mathrm{km} 0$, and they travel without any control measure until $\mathrm{km} 1$, i.e. the traffic flow is in state $A$. The generation section is present to capture any disruptions that propagate upstream created by the reduction in speed of cooperative vehicles. At $\mathrm{km} 1$ the vehicles enter the transition section, where the cooperative vehicles reduce their speed and the normal vehicles start compacting following normal car-following rules. After several tests, the transition section has been chosen long enough to ensure that all vehicles will complete the platooning process in this segment, even for the most demanding case of low flow and large platoon. This section, from $\mathrm{km}$ 1 to $\mathrm{km} \mathrm{10}$, is used to evaluate how much time and space is needed to complete the platoon formation, i.e. the transition from state $A$ to states $C$ and $O$. Finally, the control section, from $\mathrm{km} 10$ to $\mathrm{km} 11$, is used to evaluate the indexes once traffic is for certain in state $C$.

To reproduce the theoretical results shown in Figure 8, the same six indexes must be calculated from the simulation data. To understand how the indexes are calculated from simulation, an example based on a scenario with three vehicle platoons, i.e. $n_{\mathrm{p}}=3$, is presented in Figure 9 . Time, position and speed for each vehicle at each simulation time step have been recorded, and then these raw data have been elaborated to obtain the vehicle trajectories. Figure 9(a) shows an example of these trajectories, where the platoon formation is visible. Furthermore, the raw data have been manipulated to obtain the evolution of other microscopic traffic variables, e.g. spacing, headway, gap and clearance, for each vehicle during the simulation, variables necessary to re-create the theoretical results. Figure $9(b)$ shows the development of the clearance (space gap) in front of vehicles for each simulation step for the same three vehicle platoon (multiple trajectories are overlaid). The abscissa indicates the simulation step relative to the generation of the vehicle in the simulation and not the absolute simulation time; for example, the values of clearance at simulation step 200 mean that the different types of vehicles have those clearances after their positions have been updated 200 times since they entered the simulation. Because the simulation time step is equal to 0.2 second, this means that simulation step 200 is equal to 40 seconds after the vehicle generation. From the figure, it is possible to see that, due to the constant headway, every vehicle is generated with 50 metres of clearance in front of it. This initial spacing remains constant for the entire generation section, i.e. from $\mathrm{km} 0$ to $\mathrm{km} 1$, then its evolution depends on the type of vehicle and its position in the platoon. Cooperative vehicles slow down to increase the clearance in front of them until a maximum clearance is achieved (around 85 metres in this example); meanwhile the clearance in front of the following vehicles decreases to a minimum (around 25 metres), showing the change from state $A$ to state $C$ and creation of a gap $G$, i.e. state $O$. The difference between the first vehicles behind the cooperative vehicles and the second ones in the platoons is visible, with the closer vehicles reacting sooner at the reduction in speed of the cooperative vehicles, as expected. The oscillatory trajectory is due to the car-following model used that sets a limiting value for close following, around which spacing vary.

Based on these elaborated data, the six indexes have been calculated using the following procedure. The gap $G$ achievable in front of the cooperative vehicle is the average of all the gaps in front of cooperative vehicles while they are in the control section, i.e. from $\mathrm{km} 10$ to $\mathrm{km} 11$. This is because the transition section has been chosen long enough to ensure that all platoons will be completely compacted before reaching this segment.

The cooperation cycle time $C_{c}$ is calculated in the same way as in theory, Eq. (11), i.e. headway times the platoon number. Because the vehicles are generated with a constant headway, the theoretical cooperation cycle and the simulation one are identical.

Also the maximum on-ramp flow $q_{\mathrm{o}}^{\max }$, with and without the constraint of integer vehicles, is calculated in the same way as in theory. Eq. (9) states that a fixed minimum gap time for merging $g_{m}$ is necessary for each vehicle, and the same concept is used here. Therefore, the merging process is not simulated but the on-ramp flow is calculated indirectly from the size of the gap created. 


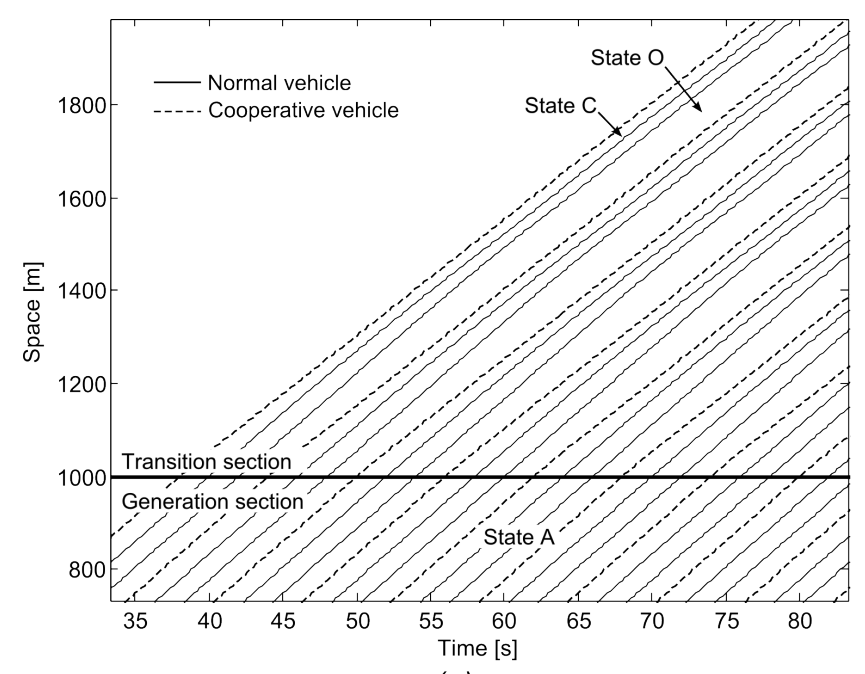

(a)

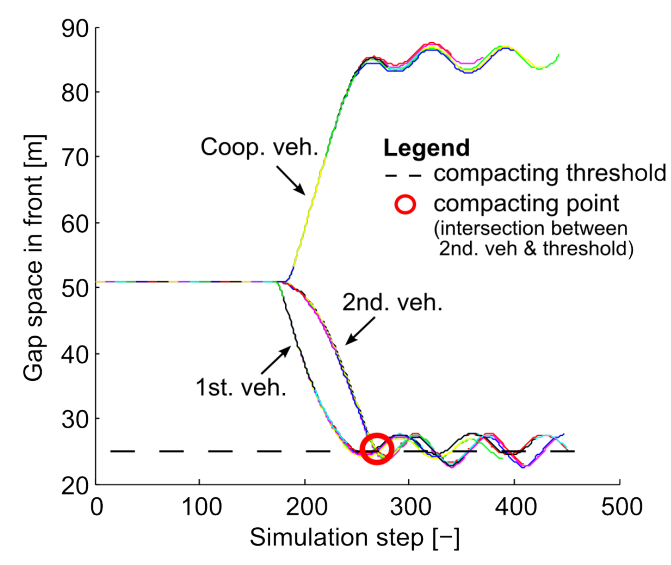

(b)

Figure 9: Control strategy validation methodology. (a) vehicle trajectories in case of a three vehicle platoon with change from state $A$, to states $C$ and $O$. (b) evolution of the clearance in front of vehicles during platoon formation for each simulation step and procedure used to calculate the indexes.

As in the case of $G$, also the space and time needed for compacting are based on the assumption that the vehicles are completely compacted within the control section. Therefore, the average spacing in this section for all the non cooperative vehicles is calculated. This value is used as a threshold to identify where and when the last vehicle in each platoon is below this threshold for the first time from the start of the cooperation. Figure $9(b)$ shows with a dashed line the value of this threshold and with a circle the point when the last vehicle in the platoon, the second vehicle behind the cooperative one in this example, is below this value. It can be see that the first vehicles reach the minimum clearance, identified by the intersection between the clearance evolution and the compacting threshold, at around simulation step 250. Instead the second vehicles reach the minimum clearance at simulation step 275, i.e. 5 seconds after the first vehicles. Then, using the trajectory data, it is possible to understand the position of the vehicles at this specific time step, and so to extract the space and time needed for compacting the platoon.

All the indexes are the averages of several cooperative vehicle cycles, but because in the scenario there is no inter-vehicle variability, no variability is present in the result either, other than some random noise introduced by the model and calculation process.

\subsection{Results and analytical vs. simulation comparison}

The theoretical results are reproduced starting from the simulation data using the described procedure. Figure 10 presents the results in analogy with Figure 8, so it is possible to refer to that image for description of the axes. From a qualitative comparison between theoretical and simulation results, it is possible to appreciate that all the six indexes present similar trends. The size of the gap $G$ for the different main carriageway flows and platoon sizes shown in Figure 10(a) follows the same evolution as in theory. $G$ decreases with the increase of $A$ and with the reduction of $n_{\mathrm{p}}$. Because the arrival of the simulated vehicles is uniform, the cooperative cycle $C_{c}$ in Figure $10(b)$ is identical for both cases as expected. Also Figure 10(c) presents the same theoretical patterns of the maximum on-ramp flow with the constraint that only an integer number of vehicles are able to merge. It should be noted that the lines indicating the loss of merging capacity due to the reduction of the number of vehicles able to merge in the gap are not perfectly vertical due to the discretization in the simulated flows. Figure 10(d) shows that smaller platoon sizes are more efficient, once again in agreement with the theoretical consideration. This behaviour is particularly important because it was obtained in theory adding the microscopic variable $g_{C}^{\mathrm{s}}$, i.e. the separation in space (clearance) between two consecutive vehicles, in Eq. (7). The same behaviour is recreated in simulation indicating that the developed theory can reproduce microscopic phenomena. Finally, also the time and space necessary for compacting the platoon, Figure 10(e) and $(f)$, present trends similar to the theoretical ones. Differently from the previous indexes, the evolution of $t_{C}$ and $x_{C}$ is not smooth, and values for different platoon sizes overlap in several cases. This phenomenon was unexpected because the simulation does not incorporate variability. A possible explanation is given by 


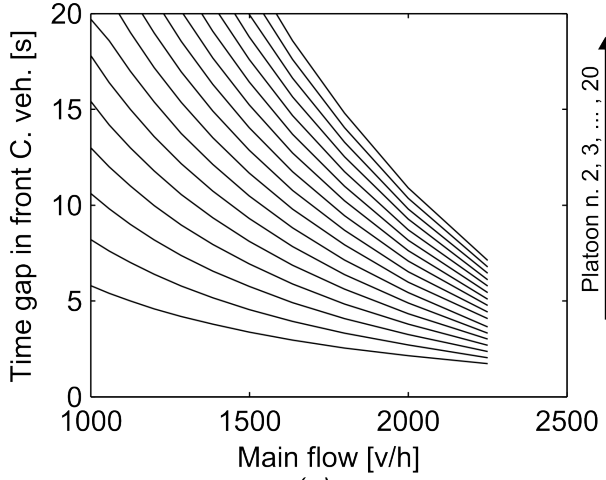

(a)

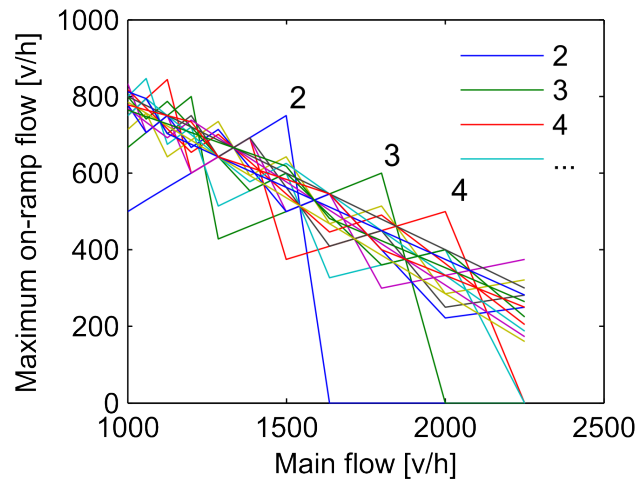

(c)

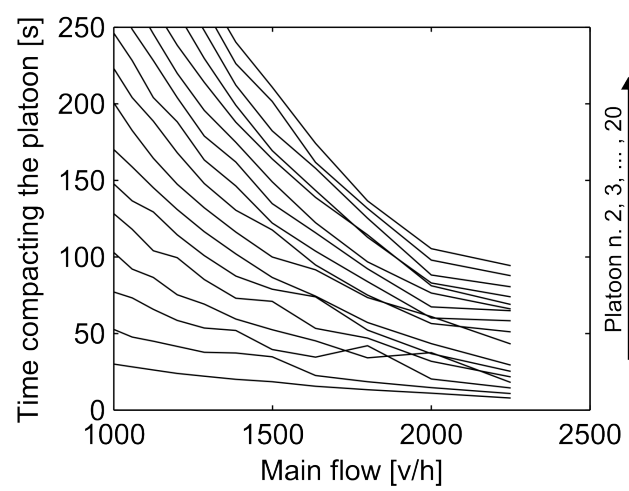

(e)

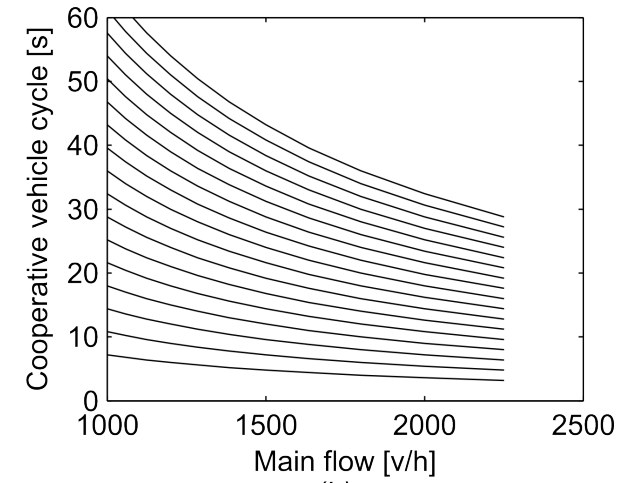

(b)

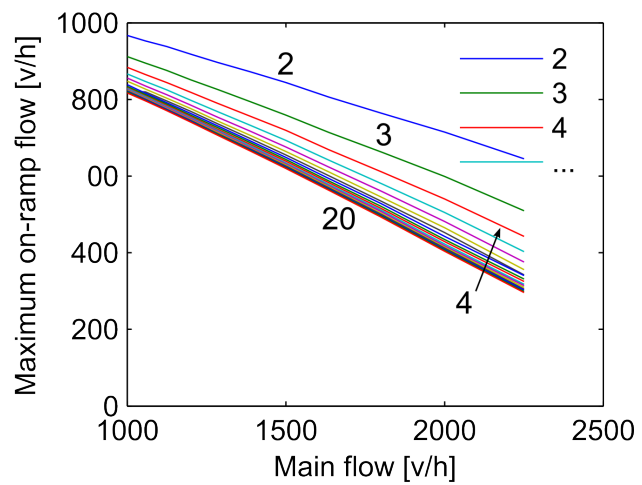

(d)

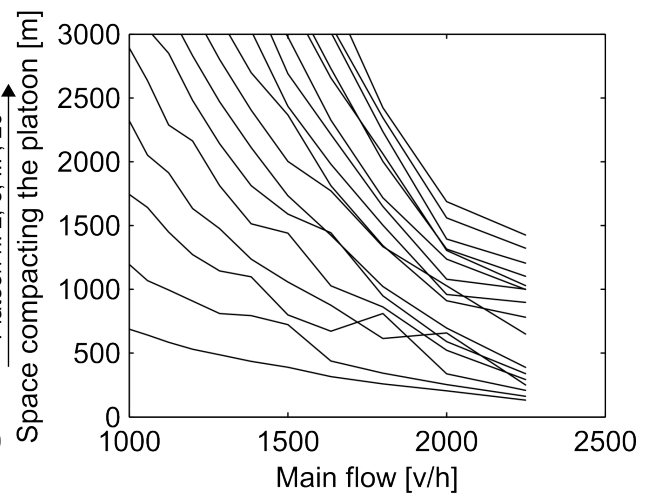

(f)

Figure 10: CoopMA formulation results obtained by simulation. (a) gap $G$ created in front of the cooperative vehicle, $(b)$ cooperative vehicle cycle $C_{c}$, (c) maximum on-ramp flow $q_{\mathrm{o}}^{\max }$ with the constraint of integer number of vehicles able to merge and $(d)$ without this constraint, $(e)$ time $t_{C}$ needed to create the gap and $(f)$ the relative space $x_{C}$. 
the small randomness introduced by VISSIM, as shown by the slightly different trajectories displayed in Figure 9(b).

Figure 11 presents the comparison between the theoretical results (abscissa), created based on a model of the fundamental diagram fitted on VISSIM data, and the simulation results (ordinate). A good agreement for all the six indexes is visible. Figure 11(a) shows that the theoretical results underestimate the size of the gap $G$ for each flow and platoon size. However this bias follows a linear behaviour. As expected the cooperation cycle is exactly the same in the two cases because the vehicles are generated with a constant headway, Figure 11(b). As a consequence of the bigger gap $G$, the simulated maximum on ramp flow is greater that the theoretical one, Figure $11(c)$, clearly visible looking at $q_{\mathrm{o}}^{\max }$ without the constraint of integer vehicles, Figure $11(d)$. Simulation and the analytical values of the time and space needed for completely compacting the platoon, Figure 11(e) and $(f)$, are once again in good agreement, proving the quality of using shock wave and individual vehicle trajectories for the estimation of the front evolutions.

The correlation coefficients between simulation and theoretical results calculated for the 6 indexes are in all cases greater than 0.9 , indicating that the analytical formulation well describes the simulated phenomena. However, as visible from Figure 11, the lines do not lie perfectly on the bisector, meaning that in each case the analytical formulation either overestimates or underestimates the indexes with a proportional error.

These errors can be reduced by adding a parameter to adjust the equations for fine tuning the analytical formulation. For example, equations 5-8 estimating $G$ as function of $A, C$ and $n_{\mathrm{p}}$, could be reformulated as:

$$
\bar{G}=\alpha \cdot G\left(A, C, n_{\mathrm{p}}\right)
$$

Where $\bar{G}$ is the value of the gap specific for a junction and $\alpha$ is a fine tuning parameter to be estimated from real observations to reduce the bias shown in Figure 11(a).

On the other hand, the discrepancy present between simulation and analytical values for the time and space needed for completely compacting the platoon can be reduced using a different approach. A safety factor could be introduced to ensure the complete transition from state $A$ to state $C$ and $O$. The time necessary for compacting the platoon suggested by the analytical formulation, i.e. $t_{C}$, could be incremented by a fixed value, with the only disadvantage of increasing the driver cooperation time. However if this increment remains in an acceptable range, for example, a few seconds, it could be easily accepted.

\section{Traffic performance evaluation}

The focus of this paper is on the definition of the control strategy equations and not on the traffic performance evaluation. Therefore, here, only a brief description of the main findings is given. A complete evaluation of the traffic performance of the Cooperative Merging Assistant system is done in Scarinci et al. (2013), Scarinci (2014) and Scarinci et al. (2015).

We use VISSIM as microscopic traffic simulation, and the CoopMA strategy is coded in MATLAB. We show that the CoopMA systemcan reduce the occurrence of congestion at junctions.

This tool has been used in recent evaluations of cooperative merging control strategies (Park et al., 2011; Marinescu et al., 2012). The default parameters have been proved to reproduce the significant traffic phenomena such as congestion creation, congestion propagation speed, break-down occurrence and location, merging process and courtesy yielding (Kerner, 2004; Cassidy and Bertini, 1999; Kim and Coifman, 2013; Daamen et al., 2010; Marczak et al., 2013)

Two scenarios are modelled:Reference - uncontrolled and Cooperative Merging Assistant - controlled by Cooperative Merging Assistant.

The motorway stretch simulated is based on a standard English motorway junction equipped with ramp metering (DfT, 2011).

Each scenario is evaluated over a range from absence of congestion to fully congested. The main carriageway flow (state $A$ ) is constant and equal to $2,000 \mathrm{veh} / \mathrm{h}$, and the on-ramp demand varies from $200 \mathrm{veh} / \mathrm{h}$ to $900 \mathrm{veh} / \mathrm{h}$. We perform 30 runs for each case with a different random seed, and we collect the distribution of the performance indexes.

We show the results of two measures of effectiveness (MoE). (i) $\gamma_{q}$ occurrence of congestion (proportion $0-1$ ), calculated as the number of single run simulations with the creation of congestion divided by the total number of single run simulations. (ii) $\lambda_{q}$ proportion of late-merging vehicles (proportion 0-1), calculated as the vehicles merging in the last 50 metres of the acceleration lane divided by the total 


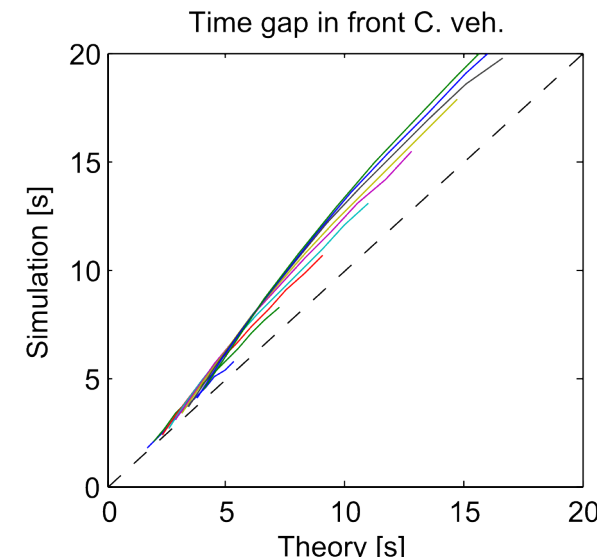

(a)

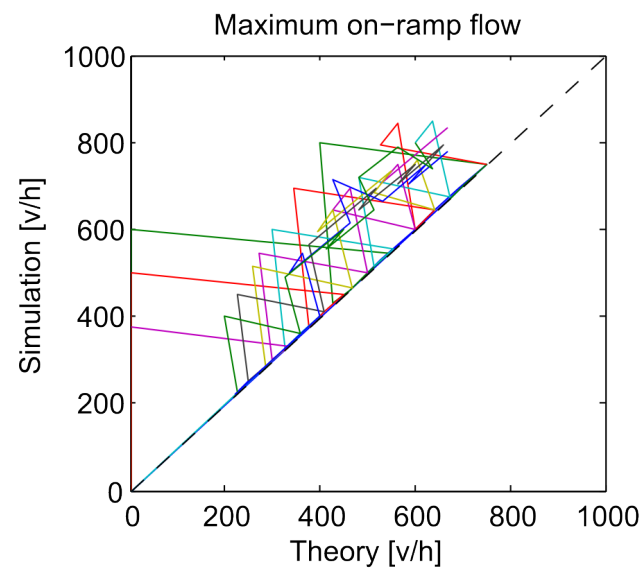

(c)

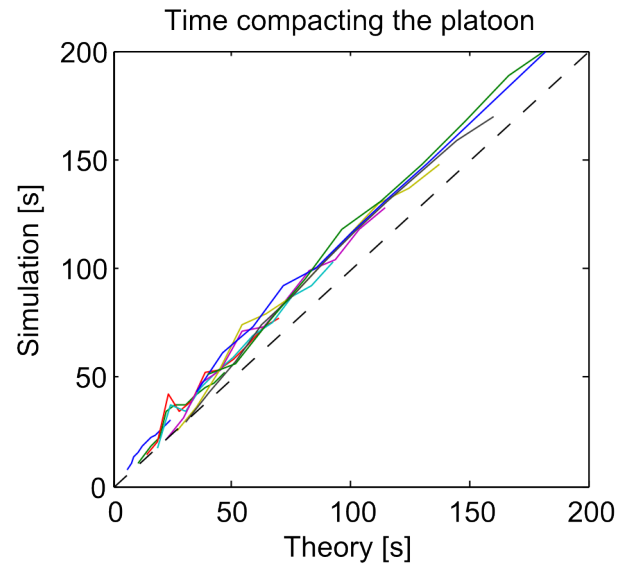

(e)

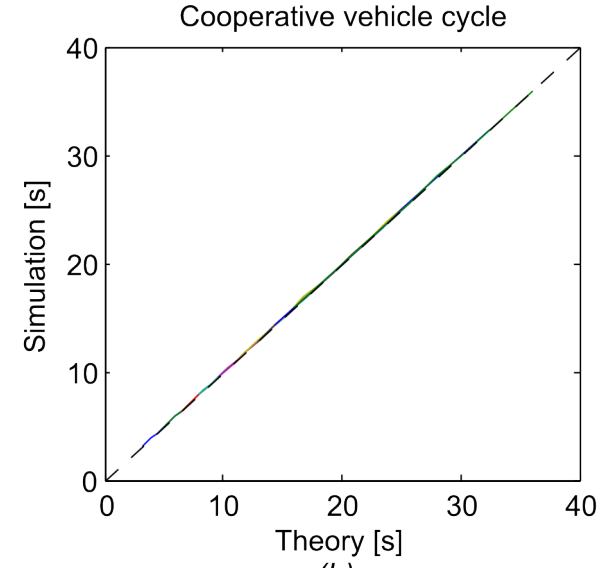

(b)

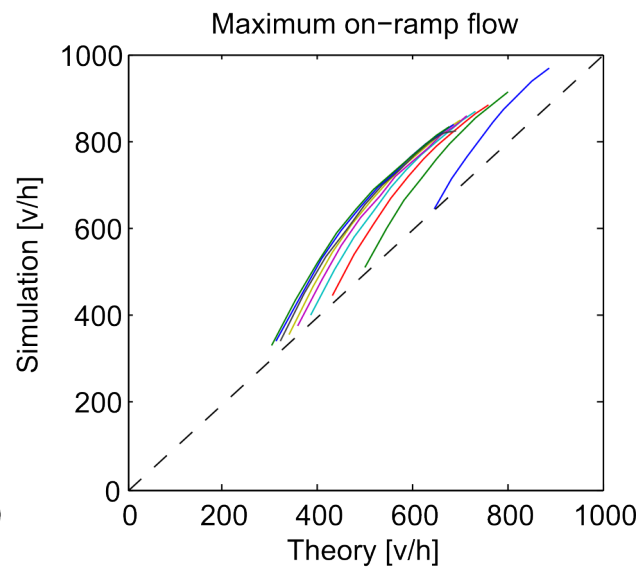

(d)

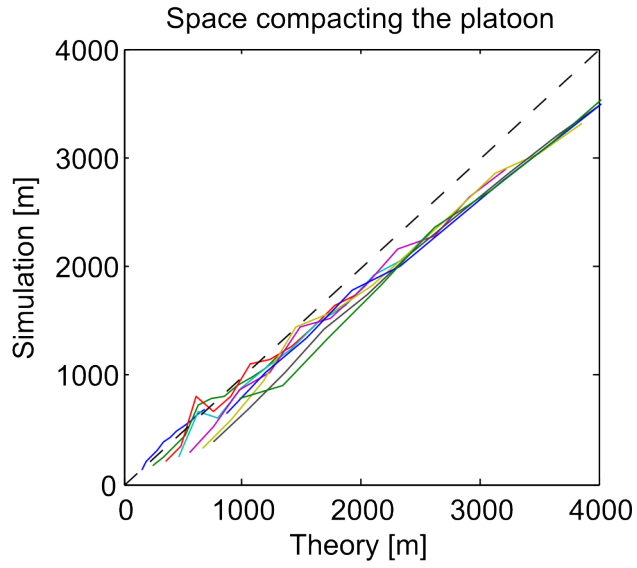

(f)

Figure 11: Comparison between theoretical and simulation results. (a) gap $G$ created in front of the cooperative vehicle, (b) cooperative vehicle cycle $C_{c},(c)$ maximum on-ramp flow $q_{\mathrm{o}}^{\max }$ with the constraint of integer number of vehicles able to merge and $(d)$ without this constraint, $(e)$ time $t_{C}$ needed to create the gap and $(f)$ the relative space $x_{C}$. 


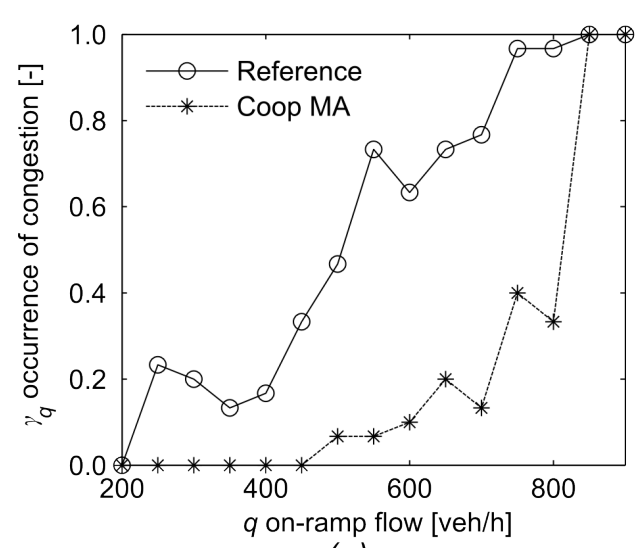

(a)

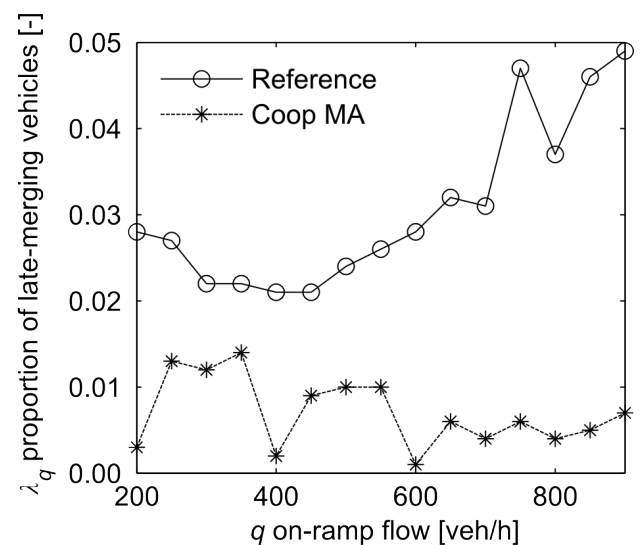

(b)

Figure 12: Simulation results showing the improvement of the traffic performance using CoopMA. (a) $\gamma_{q}$ occurrence of congestion. (b) $\lambda_{q}$ proportion of late-merging vehicles. Adapted from (Scarinci et al., 2015).

of merging vehicles.A vehicle is considered as late merging if it merges in the last 50 metres of the acceleration lane (Daamen et al., 2010), i.e. after 200 metres from the start of the merging link.

Figure 12 shows the two MoEs presented offering a graphical comparison between the uncontrolled and controlled scenarios. In Figure 12(a) is clearly visible that the use of CoopMA greatly reduces the occurrence of congestion. This result is obtained thanks to the creation of suitable gaps for merging that brings to a reduction in late-merging vehicles, as visible in Figure 12 (b), and so a decrease of disruptions that could lead to congestion. The results show that at each level of mainstream traffic, more merging traffic can be introduced with cooperative control than without it. From these simulation results, we conclude that the Cooperative Merging Assistant can improve the traffic performance.

\section{Conclusions}

In this paper, we define the Cooperative Merging Assistant control strategy using macroscopic and microscopic traffic flow theory. The analytical formulation describes the gap size for merging, traffic light cycle, maximum on-ramp flow, and time and space needed for creating the merging gap. The results evaluated for different platoon sizes and traffic conditions also suggest the practicality of the system, requesting a reasonable cooperation time from drivers and adequate on-ramp flows. The results estimated by the analytical formulation of the Cooperative Merging Assistant algorithm presented in Section 3 have been recreated using the simulation approach introduced in Section 4 . The simulation results are in good agreement with the analytical ones, and all the trends are reproduced correctly. Finally, the evaluation of the CoopeRM traffic performance has been introduced in Section 5 showing the capability of this innovative system to reduce occurrence of congestion and late-merging vehicles. Although the algorithm was developed from the state of the art theories and validated using the widely adopted commercial simulation suite VISSIM (PTV, 2013), it cannot yet be considered to be fully practice-ready. However, this paper shows the advantages of cooperative traffic management and the potential benefits of this.

An interesting development is the adaptation of the control strategy in the case of non-homogeneous gaps and mixed traffic. On motorways, vehicles normally arrive in platoons with randomly distributed gaps (Banks, 2003; Branston, 1976). To maximise the use of intra-platoon gaps or large inter-platoon gaps, a dynamic Cooperative Merging Assistant control strategy could be used. The control centre should be able to look for a spatio-temporal window in which to scan for the presence of large gaps and equipped vehicles. Then, using Eq. (2)-(21), the algorithm should set the traffic light timings accordingly. In this case, optimization could be used to identify the optimal sequence of gaps $G$ given the distribution of cooperative vehicles and constrains on the maximum length of the cooperation cycle $C_{\mathrm{c}}$.

A similar approach can be used for mixed traffic. In the near future, traffic will be composed of a mix of equipped and un-equipped vehicles, and management systems usable in this transition period should be developed. In the case of high penetration rates, it is preferable to keep a cooperation cycle $C_{\mathrm{c}}$ as constant as possible. Therefore, once identified the ideal position of the next potential cooperative vehicle, the 
algorithm should look for the closer equipped vehicle. Then, the traffic light cycle should be readjusted to coordinate the release of on-ramp vehicles accordingly. For low (or varying) penetration rates, an approach similar to the one for non-homogeneous gaps could be used. This design gives flexibility to the system allowing the release of platoon sizes defined dynamically, and so the system can be used during a transition period. The equations needed to calculate when and where control the cooperative vehicles are the same presented in Section 3.3. Therefore, the expansion to non-homogeneous and mixed traffic is based on the same theoretical foundation. The consequences on the traffic performance, which should be evaluated adequately using simulation, are left to further work.

\section{Acknowledgements}

The authors are grateful to the EC FP7 NEARCTIS project for funding this research and to the Highways Agency of England for providing access to the MIDAS data.

\section{References}

Antoniotti, M., Desphande, A., Girault, A., 1997. Microsimulation analysis of multiple merge junctions under autonomous AHS operation, in: IEEE Conference on Intelligent Transportation System ITSC, pp. $147-152$.

van Arem, B., van Driel, C.J.G., Visser, R., 2006. The Impact of Cooperative Adaptive Cruise Control on Traffic-Flow Characteristics. Intelligent Transportation Systems, IEEE Transactions on 7, 429-436.

Banks, J.H., 2003. Average time gaps in congested freeway flow. Transportation Research Part A: Policy and Practice 37, 539-554.

Branston, D., 1976. Models of single lane time headway distributions. Transportation Science 10, 125148.

Cassidy, M.J., Bertini, R.L., 1999. Some traffic features at freeway bottlenecks. Transportation Research Part B: Methodological 33, 25-42.

COOPERS, 2010. COOPERS: Co-operative systems for intelligent roard sefety. URL: www . coopers-ip. eu.

Daamen, W., van Arem, B., Bouma, I., 2011. Microscopic dynamic traffic management: simulation of two typical situations, in: 14th IEEE International Intelligent Transportation Systems Conference, ITSC, pp. 1898-1903.

Daamen, W., Loot, M., Hoogendoorn, S.P., 2010. Empirical analysis of merging behavior at freeway on-ramp. Transportation Research Record , 108-118.

Davis, L.C., 2007. Effect of adaptive cruise control systems on mixed traffic flow near an on-ramp. Physica A: Statistical Mechanics and its Applications 379, 274-290.

DfT, 2011. Design manual for roads and bridges. Department of Transport, Great Britain. ISBN 0115531742 .

FHA, 2010. Highway Capacity Manual. National Research Council. ISBN 9780309160773.

Greenshields, B.D., 1935. A Study of Traffic Capacity. Highway Research Board Proceedings 14, 448-477.

HA, 2007. Motorway Incident Detection and Automatic Signalling (MIDAS) Design Standard. 1st ed., Highways Agency, Bristol.

Hegyi, A., 2004. Model Predictive Control for Integrating Traffic Control Measures. Ph.D. thesis. Technical University of Delft.

Hegyi, A., Hoogendoorn, S.P., 2010. Dynamic speed limit control to resolve shock waves on freeways - Field test results of the SPECIALIST algorithm, in: 2010 13th International IEEE Conference on Intelligent Transportation Systems (ITSC 2010), pp. 519-24. 
Hegyi, A., Hoogendoorn, S.P., Schreuder, M., Stoelhorst, H., Viti, F., 2008. SPECIALIST: A dynamic speed limit control algorithm based on shock wave theory, in: 2008 11th International IEEE Conference on Intelligent Transportation Systems, pp. 827-32.

Kachroo, P., Li, Z., 1997. Vehicle merging control design for an automated highway system, in: IEEE Conference on Intelligent Transportation Systems, ITSC, pp. 224-229.

Kanavalli, A., Ranga, L.V.U., Sathish, A.G., Shenoy, P.D., Venugopal, K.R., Patnaik, L.M., 2008. Proactive sliding -window strategy for merging sensor-enabled cars, in: 16th IEEE International Conference on Networks, ICON, pp. 1-5.

Kato, S., Tsugawa, S., Tokuda, K., Matsui, T., Fujii, H., 2002. Vehicle control algorithms for cooperative driving with automated vehicles and intervehicle communications. Intelligent Transportation Systems, IEEE Transactions on $3,155-161$.

Kerner, B.S., 2004. The physics of traffic: empirical freeway pattern features, engineering applications, and theory. Understanding complex systems, Springer, Berlin. ISBN 3540207163.

Kerner, B.S., Rehborn, H., 1997. Experimental properties of phase transitions in traffic flow. Physical Review Letters 79, 4030.

Kim, S., Coifman, B., 2013. Driver relaxation impacts on bottleneck activation, capacity, and the fundamental relationship. Transportation Research Part C: Emerging Technologies .

Kotsialos, A., Papageorgiou, M., Hayden, J., Higginson, R., McCabe, K., Rayman, N., 2006. Discrete release rate impact on ramp metering performance. IEE Proceedings: Intelligent Transport Systems 153, 85-96.

Leclercq, L., Laval, J.A., Chiabaut, N., 2011. Capacity drops at merges: An endogenous model. Transportation Research Part B: Methodological 45, 1302-1313.

Lighthill, M.J., Whitham, G.B., 1955. On kinematic waves. II. A theory of traffic flow on long crowded roads. Proceedings of Royal Society 229A, 317-345.

Lu, X.Y., Tan, H., Shladover, S.E., Hedrick, J.K., 2004. Automated Vehicle Merging Maneuver Implementation for AHS. Vehicle System Dynamics 41, 85-107.

Mammano, F.J., Bishop, J.R., 1992. Status of IVHS technical developments in the United States, in: 42nd IEEE Vehicular Technology Conference, pp. 85-88.

Marczak, F., Daamen, W., Buisson, C., 2013. Key Variables of Merging Behaviour: Empirical Comparison between Two Sites and Assessment of Gap Acceptance Theory. Procedia-Social and Behavioral Sciences 80, 678-697.

Marinescu, D., Curn, J., Bouroche, M., Cahill, V., 2012. On-ramp traffic merging using cooperative intelligent vehicles: A slot-based approach, in: 15th International IEEE Conference on Intelligent Transportation Systems, ITSC, pp. 900-906.

Marinescu, D., Curn, J., Slot, M., Bouroche, M., Cahill, V., 2010. An active approach to guaranteed arrival times based on traffic shaping, in: 13th International IEEE Conference on Intelligent Transportation Systems, ITSC, pp. 1711-1717.

MathWorks, 2013. MATLAB. URL: www.mathworks.com.

NEARCTIS, 2009. Deliverable 6. Preliminary Report on the review of the state of knowledge and capability in relevant technologies. Technical Report. Nearctis Consortium, European Commission.

Netten, B., Wilmink, I., Hogema, J., Hegyi, A., Wang, M., Baan, J., Passchier, I., 2011. Dynamax In Car: system concepts, research questions, hypotheses, simulation and evaluation. Technical Report DV 2011 C474. TNO: Netherlands Organisation for Applied Scientific Research.

Ntousakis, I.A., Nikolos, I.K., Papageorgiou, M., 2016. Optimal vehicle trajectory planning in the context of cooperative merging on highways. Transportation Research Part C: Emerging Technologies 71, 464-488. 
Papageorgiou, M., Kotsialos, A., 2002. Freeway Ramp Metering: an overview. IEEE Transactions on Intelligent Transportation Systems 3, 271-281.

Papageorgiou, M., Papamichail, I., 2008. Overview of traffic signal operation policies for ramp metering. Transportation Research Record , 28-36.

Park, H., Bhamidipati, C.S., Smith, B.L., 2011. Development and evaluation of enhanced intellidriveenabled lane changing advisory algorithm to address freeway merge conflict. Transportation Research Record , 146-157.

Prigogine, I., Herman, R., 1971. Kinetic theory of vehicular traffic. American Elsevier Pub. Co. ISBN 9780444000828 .

PTV, 2013. VISSIM. URL: http://vision-traffic.ptvgroup.com.

Pueboobpaphan, R., Liu, F., van Arem, B., 2010. The impacts of a communication based merging assistant on traffic flows of manual and equipped vehicles at an on-ramp using traffic flow simulation, in: 13th International IEEE Conference on Intelligent Transportation Systems, ITSC, pp. 1468-1473.

Ran, B., Leight, S., Chang, B., 1999. A microscopic simulation model for merging control on a dedicatedlane automated highway system. Transportation Research Part C: Emerging Technologies 7, 369-388.

Ranjitkar, P., Nakatsuji, T., Azuta, Y., Gurusinghe, G.S., 2003. Stability analysis based on instantaneous driving behavior using car-following data. Transportation Research Record: Journal of the Transportation Research Board 1852, 140-151.

Scarinci, R., 2014. Managing traffic at motorway junctions: a ramp metering development using intelligent vehicles. Ph.D. thesis. University College London UCL.

Scarinci, R., Heydecker, B., Hegyi, A., 2015. Analysis of Traffic Performance of a Merging Assistant Strategy Using Cooperative Vehicles. IEEE Transactions on Intelligent Transportation Systems 16, 2094-2103.

Scarinci, R., Heydecker, B.G., 2014. Control concepts for facilitating motorway on-ramp merging using intelligent vehicles. Transport Reviews 34, 775-797.

Scarinci, R., Heydecker, B.G., Hegyi, A., 2013. Analysis of Traffic Performance of a Ramp Metering Strategy Using Cooperative Vehicles, in: 16th International IEEE Conference on Intelligent Transportation Systems - ITSC.

Uno, A., Sakaguchi, T., Tsugawa, S., 1999. Merging control algorithm based on inter-vehicle communication, in: IEEE International Conference on Intelligent Transportation Systems, pp. 783-787.

Wang, Z., Kulik, L., Ramamohanarao, K., 2007. Proactive traffic merging strategies for sensor-enabled cars, in: VANET'07: Fourth ACM International Workshop on Vehicular Ad Hoc Networks, pp. 39-48.

Wiedemann, R., 1974. Simulation des Strassenverkehrsflusses. Technical Report. Institute for Traffic Engineering, University of Karlsruhe.

Xie, Y., Zhang, H., Gartner, N.H., Arsava, T., 2016. Collaborative merging strategy for freeway ramp operations in a connected and autonomous vehicles environment. Journal of Intelligent Transportation Systems , 1-12.

Xu, Q., Sengupta, R., 2003. Simulation, analysis, and comparison of ACC and CACC in highway merging control, in: Intelligent Vehicles Symposium IEEE proceedings, pp. 237-242.

Yang, C., Kurami, K., 1993. Longitudinal guidance and control for the entry of vehicles onto automated highways, in: 32nd IEEE Conference on Decision and Control, p. 1891.

Yang, C., Milacic, M., Kurami, K., 1993. A Longitudinal Control Concept For Merging Of Automated Vehicles, in: Intelligent Vehicles Symposium, pp. 408-413.

Zheng, P., McDonald, M., 2007. Evaluation of effects of ramp metering on merging operations. Transportation Research Record, 105-112. 